\title{
Multiple genetic pathways regulating lifespan extension are neuroprotective in a G2019S LRRK2 nematode model of Parkinson's disease
}

Megan M. Senchuk ${ }^{1}$, Jeremy M. Van Raamsdonk ${ }^{2,3,4, *}$ and Darren J. Moore ${ }^{1, *}$

${ }^{1}$ Center for Neurodegenerative Science, Van Andel Institute, Grand Rapids, MI 49503, USA

${ }^{2}$ Department of Neurology and Neurosurgery, McGill University, Montreal, Quebec, H4A 3J1, Canada

${ }^{3}$ Metabolic Disorders and Complications Program, and Brain Repair and Integrative Neuroscience Program, Research Institute of the McGill University Health Centre, Montreal, Quebec, H4A 3J1, Canada

${ }^{4}$ Department of Genetics, Harvard Medical School, Cambridge, MA 02115, USA

${ }^{*}$ Co-corresponding authors:

Darren Moore (darren.moore@vai.org)

Jeremy Van Raamsdonk (jeremy.vanraamsdonk@mcgill.ca) 


\section{Abstract}

Background: Mutations in the leucine-rich repeat kinase 2 (LRRK2) gene are the most frequent cause of late-onset, familial Parkinson's disease (PD), and LRRK2 variants are associated with increased risk for sporadic PD. While advanced age represents the strongest risk factor for disease development, it remains unclear how different age-related pathways interact to regulate LRRK2-driven late-onset PD.

Findings: In this study, we employ a C.elegans model expressing PD-linked G2019S LRRK2 to examine the interplay between age-related pathways and LRRK2-induced dopaminergic neurodegeneration. We find that multiple genetic pathways that regulate lifespan extension can provide robust neuroprotection against mutant LRRK2. However, the level of neuroprotection does not strictly correlate with the magnitude of lifespan extension, suggesting that lifespan can be experimentally dissociated from neuroprotection. Using tissue-specific RNAi, we demonstrate that lifespan-regulating pathways, including insulin/IGF-1 signaling, TOR, and mitochondrial respiration, can be directly manipulated in neurons to mediate neuroprotection. We extend this finding for AGE-1/PI3K, where pan-neuronal versus dopaminergic neuronal restoration of AGE-1 reveals both cell-autonomous and non-cell-autonomous neuroprotective mechanisms downstream of insulin signaling.

Conclusions: Our data demonstrate the importance of distinct lifespan-regulating pathways in the pathogenesis of $L R R K 2$-linked PD, and suggest that extended longevity is broadly neuroprotective via the actions of these pathways at least in part within neurons. This study further highlights the complex interplay that occurs between cells and tissues during organismal aging and disease manifestation. 


\section{Background}

Parkinson's disease (PD) is a common neurodegenerative disorder, affecting 1-2\% of individuals over 65 years of age (de Lau and Breteler, 2006). With population demographics predicting an increasingly elderly population, the prevalence of PD is expected to grow to over 12 million cases by 2040 (GBD 2016 Neurology Collaborators, 2019; Dorsey et al, 2018). PD is a progressive movement disorder characterized by resting tremor, muscular rigidity, and bradykinesia primarily resulting from the selective degeneration of dopaminergic neurons in the substantia nigra and the corresponding loss of nigrostriatal pathway dopamine signaling. While PD is influenced by both genetic risk and environmental exposure, the etiology of PD remains unclear. Aging is considered a primary risk factor for the development of PD, as the incidence of disease rises exponentially with increasing age (Billingsley et al, 2018). Aging is associated with the impairment of numerous cellular pathways, including mitochondrial respiration, proteostasis, autophagy and stress responses that lead to a reduced chaperone response and increased genomic instability. A number of pathways have been identified that can regulate chronological age yet the relationship between these specific lifespan-regulating pathways and pathogenic mechanisms underlying PD are poorly understood.

The identification of genetic mutations linked to familial forms of PD have provided important insight into disease pathophysiology. Mutations in leucine-rich repeat kinase 2 (LRRK2) cause late-onset, autosomal dominant familial PD that is largely indistinguishable from sporadic disease, whereas both coding and non-coding variants are associated with an increased risk of sporadic PD (Hernandez et al, 2016). LRRK2 encodes a multidomain protein containing kinase, GTPase, and protein-protein interaction domains, with familial mutations commonly leading to enhanced kinase activity. The biological functions of LRRK2 remain to be fully elucidated, although prior studies suggest roles in diverse cellular processes, including vesicular transport/sorting, endocytosis, autophagy, mitochondrial dynamics, and lysosomal function. Familial LRRK2 mutations share the capacity to induce neuronal damage in culture models via a kinasedependent mechanism, whereas increased LRRK2 kinase activity has been reported in PD-linked D620N VPS35 knockin mice and sporadic PD brain. LRRK2 kinase activation may therefore represent a common mechanism underlying PD pathogenesis (Kluss et al, 2019), and a promising target for therapeutic disease-modification.

Among familial LRRK2 mutations, G2019S is the most common, accounting for $5-6 \%$ of familial PD and 1-2\% of sporadic PD in the US population (Billingsley et al, 2018). PD linked to the G2019S mutation is typically late-onset and exhibits variable yet age-dependent penetrance depending on ethnicity, with estimates of cumulative risk rising from $\sim 20 \%$ at 50 years to $\sim 75 \%$ 
at 80 years of age (Spatola and Wider, 2014). The exponential rise in PD risk after age 50 suggests that the aging process may play an important role in manifesting the pathogenic actions of LRRK2 mutations (Marder et al, 2015). This interaction of age with LRRK2 is borne out in experimental models, such as G2019S LRRK2 knockin or transgenic mice, Drosophila or C.elegans, that develop age-dependent and often late-onset PD-like phenotypes (Longo et al, 2017; Ho et al, 2019; Xiong et al, 2017). Some individuals harboring LRRK2 mutations escape PD within their lifetime, suggesting that genetic variation within different age-related pathways might feasibly be protective and could be manipulated to prevent disease.

While incomplete penetrance suggests that age-related pathways significantly interact with G2019S LRRK2 during disease progression, experimental mammalian models including human induced pluripotent stem cells and rodent models are cost-prohibitive or not practical for chronic aging studies. We therefore took advantage of the nematode Caenorhabditis elegans, a wellestablished model for aging studies, to systematically investigate functional interactions between age-related pathways and G2019S LRRK2-induced dopaminergic neurodegeneration. Conserved signaling pathways regulating the aging process include insulin/insulin-like growth factor 1 (IGF-1) signaling, target of rapamycin (TOR) pathway, signals from the reproductive system, environmental sensing, and caloric restriction. These pathways define major nutrientsensing, endocrine, and stress response signaling pathways, and are required for the coordinated regulation of systemic organismal aging.

Our recent study demonstrated that reducing insulin/IGF-1 signaling, a well-characterized pathway for regulating lifespan, increased dopaminergic viability in C.elegans models of PD expressing human mutant LRRK2 or a-synuclein (Cooper et al, 2015). However, the underlying mechanism for this neuroprotective effect is not clear. It is possible that insulin/IGF-1 signaling interacts directly with G2019S LRRK2 to attenuate neurodegenerative phenotypes, and would therefore represent a potential therapeutic pathway. Alternatively, reduced insulin signaling may act indirectly by slowing the rate of aging in all tissues, and this feature may regulate LRRK2induced neurotoxicity. To distinguish between lifespan extension in general versus the insulin signaling pathway as a key neuroprotective mechanism, we evaluate and compare the neuroprotective effects of multiple distinct lifespan-extending pathways in the G2019S LRRK2 worm model. Our data demonstrate that lifespan extension via multiple signaling pathways can regulate dopaminergic neuronal resilience towards G2019S LRRK2.

\section{Results}




\section{G2019S LRRK2 induces age-dependent dopaminergic neuronal damage but does not} influence lifespan.

This study seeks to explore the role of different age-related pathways in regulating pathogenic phenotypes in a G2019S LRRK2 worm model of PD. We have employed a transgenic model expressing full-length human G2019S LRRK2 under the control of the presynaptic dopamine transporter promoter (Dat-1). A pDat-1::GFP transgene expressed in cis serves as a method to visualize the eight dopaminergic neurons in C.elegans from a total of $\sim 302$ neurons. Previous studies have demonstrated that this model shows reduced dopaminergic neuronal function as indicated by subtle movement phenotypes and age-related changes in dopaminergic neurons monitored using pDat-1::GFP (Yao et al, 2010, Cooper al al, 2015). The pDat1::hLRRK2(G2019S); pDat-1::GFP line (cwrls856) is hereafter referred to as LRRK2(G2019S).

Our study focuses primarily on age-dependent neurodegenerative changes within the four anterior cephalic dopaminergic neurons (CEPs), as these neurons are relatively large in size with extended axonal processes and are simple to distinguish from intestinal autofluorescence (lipofusucin accumulation). Our initial validation of this model reveals that CEP neurons expressing LRRK2(G2019S) remain morphologically normal up to adult day 7 (Figure 1A). By adult day 9, large GFP-positive puncta are observed within and proximal to the CEP neuronal soma. By day 12, GFP-positive puncta are observed throughout the axon and dendrites, and GFP intensity within processes is reduced. Changes in cell soma morphology (i.e. swelling, reduced circularity) are also observed. As organismal aging continues to day 15, axonal processes become increasingly disorganized, and GFP intensity in the cell soma is greatly reduced. GFP appears to coalesce in puncta within the axon, as these remain bright despite the loss of GFP within the cell soma and dendritic processes. The GFP-positive puncta are consistent with the formation of axonal spheroids or inclusions that are commonly observed in degenerating neurons.

Based on these initial observations, we developed a robust scoring metric graded from 0 (normal) to 5 (maximum damage) to quantify overall damage in the four CEP dopaminergic neurons that incorporates measures of relative intensity of GFP, the formation of GFP-positive puncta in cell soma and processes, abnormal cell soma morphology (swelling, reduced circularity) and the disorganization of neuronal processes (Figure 1B). To further validate this scoring metric, we compare worms expressing pDat-1::GFP alone or together with LRRK2(G2019S) at days 9, 12 and 15 revealing that LRRK2(G2019S) expression induces pronounced dopaminergic neuronal damage at early time points with the accumulation of damage in an age-dependent manner compared to GFP alone (Figure 1C). A more modest reduction in GFP intensity and increased axonal disorganization are observed during aging in pDAT-1::GFP and pDAT-1::GFP; 
pDat-1::hLRRK2(WT) control lines compared to LRRK2(G2019S) (Figure 1C) (data not shown). However, GFP-positive puncta are never observed in these control lines, indicating that this phenotype is specific to LRRK2(G2019S). Despite dopaminergic neuronal damage induced by LRRK2(G2019S) expression, we observe that lifespan in this strain is not significantly altered compared to pDat-1::GFP or wild-type (non-transgenic N2) worms (Figure 1D-E), as observed previously (Yao et al, 2010; Cooper et al, 2015). Together, our data suggest that G2019S LRRK2 robustly induces age-dependent dopaminergic neuronal damage but without effects on lifespan in this worm model.

\section{Lifespan extension of LRRK2(G2019S) worms by genetic reduction of multiple negative regulators of longevity}

To determine whether G2019S LRRK2-mediated neurodegeneration is impacted by alternative modes of lifespan extension, the LRRK2(G2019S) model was crossed to a well-characterized panel of genetic mutants known to extend lifespan (negative regulators of longevity, Table 1), including: daf-2, the insulin/IGF-1 receptor (Kenyon et al, 1993; Kimura et al, 1997); age-1 (PI3K) (Friedman \& Johnson, 1988), a downstream effector kinase in the insulin/IGF-1 signaling pathway; nuo-6, a mitochondrial complex I subunit (Yang \& Hekimi, 2010); glp-I (NotchR), required for germline proliferation (Arantes-Oliveira et al, 2002); eat-2, a genetic model of caloric restriction (Lakowski \& Hekimi, 1998); rsks-1 (S6K), a downstream effector kinase of TOR signaling to regulate protein synthesis (Pan et al, 2007; Hansen et al, 2007); ife-2 (elF4E), a translation initiation factor (Hansen et al, 2007), and osm-5, an intraflagellar transport protein mediating chemosensation (Apfeld \& Kenyon, 1999). Initially, we sought to confirm that lifespan would be extended in all mutant backgrounds. On a wild-type background, LRRK2(G2019S) transgenic worms exhibit a normal lifespan with an average of $\sim 17$ days at $20^{\circ} \mathrm{C}$ similar to control wild-type (N2) worms (Figure 1D-E, Figure 2) (Yao et al, 2001; Cooper et al, 2015). When crossed into lifespan-extending mutant backgrounds, the mean lifespan of LRRK2(G2019S) worms is increased to a similar extent compared to each genetic mutant alone (Figure 2 and Table 1). Genetic mutants suppressing insulin/IGF-1 signaling (daf-2 [e1370] and age-1 [hx546]) have the strongest effect on lifespan extension, increasing mean lifespan to 39.1 days and 33.4 days of adulthood, respectively (Figure 2I). Lifespan is extended to a mean of 29.6 days in a glp-1 mutant lacking germ cells, while mitochondrial respiration (nuo-6), TOR signaling (rsks-1), caloric intake (eat-2), and elF4E-mediated protein translation (ife-2) mutants extend normal lifespan by $\sim 9$ days over LRRK2(G2019S) alone or a wild-type N2 strain (Figure 2I). Reduction of chemosensory perception (osm-5) results in a more modest $\sim 20 \%$ increase in lifespan relative to control worms 
(Figure 2I). Overall, these data demonstrate that lifespan can be successfully extended using a diverse panel of genetic mutants in LRRK2(G2019S) transgenic worms similar to non-transgenic control worms.

\section{Lifespan-extending pathways provide neuroprotection against dopaminergic neuronal damage in LRRK2(G2019S) worms}

We next assessed the extent to which different longevity pathways interact with LRRK2(G2019S)induced neurotoxicity by monitoring damage to GFP-positive dopaminergic CEP neurons, comparing LRRK2(G2019S) transgenic worms on a wild-type background to lifespan-extending mutant backgrounds under identical conditions. Using the scoring metric validated in Figure 1, dopaminergic neuronal damage was assessed at day 14 of adulthood, a timepoint where degeneration is clearly observed but neurons largely remain intact. Intriguingly, we find that all pathways of lifespan extension confer variable neuroprotection in the LRRK2(G2019S) model with reduced scores of dopaminergic neuronal damage (Figure 3). In each of the eight lifespanextending paradigms evaluated, CEP neuronal soma and axonal processes appear visibly healthier and brighter, with wider continuous processes that have increased GFP intensity and reduced GFP-positive puncta (Figure 3A). However, GFP-positive puncta are still detected in each mutant background, albeit at reduced levels, perhaps suggesting that mechanisms directly mediating LRRK2-induced toxicity are only partly impacted, and that overall organismal health is maintained for an extended period. Interestingly, we note that the degree of neuroprotection afforded by each mutant does not directly correlate with the degree of lifespan extension (Figure $2 \mathrm{I}$ and $3 \mathrm{~B}$ ). Linear regression analysis confirms the general trend that the extent of lifespan extension negatively correlates with degree of dopaminergic damage, as best exemplified by the opposing effects of daf-2 and osm-5 (Figure 3C). Certain lifespan-extending mutants, however, reveal that dopaminergic neuroprotection can be uncoupled from lifespan extension, resulting in an $\mathrm{R}^{2}$ value of 0.427 . For example, nuo- 6 displays a moderate increase in lifespan yet shows pronounced reduction of dopaminergic damage, and likewise age-1 shows robust lifespan extension yet a corresponding smaller degree of neuroprotection (Figure 3C). Collectively, these data demonstrate that delaying aging via multiple distinct pathways is broadly neuroprotective against G2019S LRRK2-induced neurotoxicity, yet lifespan extension does not strictly correlate with the magnitude of neuroprotection. Our data suggest that age-related pathways may functionally interact with G2019S LRRK2-dependent pathways to differing extents, specifically insulin signaling (daf-2), mitochondrial respiration (nuo-6) and TOR signaling (rsks-1), to mediate robust neuroprotection. 


\section{Neuroprotective effects of age-1 in the LRRK2(G2019S) model require DAF-16/FOXO}

Neuronal health of LRRK2(G2019S)-expressing dopaminergic neurons is preserved in both the daf-2 and age-1 lifespan-extending backgrounds. AGE-1/PI3K functions as a downstream effector of the insulin receptor DAF-2 to control nuclear localization of the master transcriptional regulator DAF-16/FOXO. DAF-16/FOXO functions as a downstream factor required for a number of lifespan-regulating pathways, including nutrient and stress-related pathways (Insulin/IGF-1, TOR, caloric restriction, AMPK, JNK) as well as germline signaling. Accordingly, the ability of daf2 and age-1 to increase lifespan are dependent on DAF-16 (Tissenbaum, 2018). We previously demonstrated that the protective effects of daf-2 in LRRK2(G2019S) worms was dependent on the presence of DAF-16 (Cooper et al, 2015). We confirm that lifespan extension and dopaminergic neuroprotection in LRRK2(G2019S) worms conferred by age-1(hx546) similarly requires DAF-16 function by crossing age-1;LRRK2(G2019S) worms into a daf-16 (mu86) mutant background (Figure 4). The introduction of daf-16 effectively and fully reverses the increased lifespan (Figure 4A-B) and dopaminergic protective effects (Figure 4C) mediated by age-1. As the rate of aging is increased in worms with a daf-16 mutation, dopaminergic damage was scored at day 10 as opposed to day 14 . These data demonstrate that age-1 and daf-2 similarly require the canonical insulin/IGF-1 signaling pathway functioning upstream of DAF-16/FOXO for mediating neuroprotection in LRRK2(G2019S) transgenic worms.

\section{Lifespan-extending pathways mediate neuroprotection against G2019S LRRK2 in part via cell-autonomous effects in neurons}

LRRK2(G2019S) is specifically expressed in dopaminergic neurons under the control of the Dat1 promoter with damage limited to these neurons. The aging process requires the coordination of inputs from various tissues including the intestine, neurons and germline; input signaling can be perceived by one tissue but must be relayed to and acted upon by distal tissues. It is unclear whether lifespan-extending pathways mediate dopaminergic neuroprotection in LRRK2(G2019S) worms via their direct actions in neurons or indirectly via signals derived from peripheral tissues and organs. To address this question, we performed tissue-specific RNAi-mediated silencing of key lifespan-extending genes to compare the pan-neuronal versus body-wide effects of these pathways on lifespan-extension and neuroprotection against LRRK2(G2019S). We employed an RNAi targeting system using a dsRNA uptake channel SID-1 mutant (Systemic RNAi Deficient, sid-1[qt9]) combined with a rescuing SID-1 transgene driven by the pan-neuronal Unc-119 promoter (Calixto et al, 2010). In this system, neurons expressing the SID-1 transgene are 
selectively sensitized to RNAi versus non-neuronal cells that lack SID-1. Body-wide or systemic RNAi in a wild-type background has limited impact on gene expression in neurons as prior studies have suggested that C.elegans neurons are resistant to RNAi (Kamath et al, 2001; Timmons et al, 2001; Asikainen et al, 2005). The pDat-1::LRRK2(G2019S); pDat-1::GFP transgene was crossed into the neuronal-sensitizing pUnc-119::SID-1; sid-1(qt9) background, referred to hereafter as LRRK2(G2019S); SID-1. Relative to systemic body-wide RNAi in the LRRK2(G2019S) line, neuronal-specific RNAi in the LRRK2(G2019S); SID-1 background reveals a modest reduction in mean lifespan with control RNAi (empty vector) (Figure 5A-B).

Bacterial feeding clones from the Ahringer RNAi knockout library targeting lifespan-regulating pathways were verified by sequencing and first confirmed for lifespan extension phenotypes under systemic knockdown conditions prior to studies comparing systemic and neuron-specific RNAi. Clones for eat-2 and osm-5 were not available in the RNAi library. Instead, clones targeting related members of the chemoperception pathway (che-3 and osm-6) were tested, but neither extended lifespan following RNAi-mediated knockdown and were not tested further (Figure S1). We find that the LRRK2(G2019S) [systemic RNAi] and LRRK2(G2019S); SID-1 [neuron-specific RNAi] backgrounds both exhibit marked lifespan extension in response to RNAi targeting the insulin/IGF-1 pathway (daf-2, age-1) (Figures 5A-B and S2). Surprisingly, neuron-specific RNAi to age-1 results in a 1.46-fold increase in lifespan similar to systemic RNAi (1.35-fold), suggesting that the lifespan-regulating functions of $A G E-1 / P I 3 K$ are mediated primarily via the neuronal population. Interestingly, RNAi for daf-2 demonstrates a stronger increase in lifespan with systemic (1.23-fold) compared to neuronal (1.16-fold) knockdown. These data are in agreement with previous studies demonstrating that pan-neuronal restoration of insulin/IGF-1 function partially rescues extended lifespan in mutant backgrounds (Wolkow et al, 2000; Iser et al, 2007). Importantly, the dopaminergic degenerative phenotype in LRRK2(G2019S) worms is suppressed by systemic or neuronal RNAi for age-1 and daf-2 (Figure 5C-D), similar to data obtained with genetic mutants (Figure 3), thereby suggesting that the insulin/IGF-1 signaling pathway can interact with aging and neurodegeneration via both cell autonomous and non-cell autonomous functions.

Similar to the insulin/IGF-1 pathway, targeting rsks-1 by systemic or neuronal-specific RNAi induces lifespan extension (Figure $5 A-B$ and $S 2$ ), suggesting longevity-regulating functions can occur in both neuronal and non-neuronal tissues. The pan-neuronal or systemic reduction of $r s k s-$ 1 function reduces dopaminergic neuronal damage in LRRK2(G2019S) worms to a similar extent (Figure 5C-D), although TOR pathway signaling has a greater impact on lifespan in peripheral tissues (1.2-fold) versus neurons (1.1-fold). Systemic RNAi for nuo-6 results in early larval arrest 
and embryonic lethality (Figure 5A and S2), as observed previously (Yang et al, 2010; Sonnichsen et al, 2005; Rual et al, 2004; Fraser et al, 2000), that precludes further analysis. However, neuronal-specific RNAi for nuo-6 results in modest lifespan extension (Figure 5B and S2) yet a marked reduction in dopaminergic neuronal damage (Figure 5D) in LRRK2(G2019S) worms. These data suggest a direct role for mitochondrial respiration in LRRK2(G2019S)-induced dopaminergic phenotypes that can be uncoupled from mechanisms associated with lifespan extension induced by mitochondrial dysfunction. In contrast, systemic or neuronal-specific knockdown of ife-2 induces marked lifespan extension (Figure 5A-B and S2) but fails to protect against G2019S LRRK2-induced dopaminergic damage (Figure 5C-D), demonstrating that neuroprotection does not strictly correlate with extended longevity. Collectively, our data suggest that age-related pathways can directly function in part within neurons (age-1, daf-2, nuo-6, rsks1) to mediate lifespan extension and dopaminergic neuroprotection in LRRK2(G2019S) worms.

\section{Restoration of AGE-1/PI3K in neurons differentially modulates lifespan and dopaminergic neuronal damage in LRRK2(G2019S); age-1 worms.}

Using tissue-specific RNAi, we demonstrate that a number of lifespan-regulating pathways mediate systemic effects on longevity via signaling occurring within neuronal populations, as well as exerting lifespan-regulating effects in peripheral non-neuronal tissues and cells. It is possible that reduced LRRK2(G2019S)-induced dopaminergic damage observed in lifespan-extending conditions can be attributed to systemic pathways regulating organismal aging, as both insulin/IGF-1 signaling and mitochondrial dysfunction can function via non-cell-autonomous mechanisms (Wolkow et al, 2000; Iser et al 2007; Libina et al, 2003; Zhang et al, 2013; Apfeld \& Kenyon 1998). Alternatively, signaling could be required within specific cells or populations to directly regulate their viability and health. To address whether insulin/IGF-1 signaling functions specifically within dopaminergic neurons to impact LRRK2(G2019S)-induced neurotoxicity, we compared the extent of neuroprotection in LRRK2(G2019S); age-1 worms with restoration of AGE-1 specifically targeted to dopaminergic neurons or pan-neuronally. We generated extrachromosomal transgenic arrays that drive expression of AGE-1/PI3K cDNA under the control of the pan-neuronal Unc-119 promoter or the dopaminergic-specific Dat-1 promoter, that were crossed into long-lived LRRK2(G2019S); age-1(hx546) worms for rescue studies. We elected to focus on the AGE-1/PI3K pathway for these studies since RNAi targeting of age-1 in neurons produces robust effects on both lifespan and neuroprotection compared to other pathways suggesting a prominent role in neurons (Figure 5). In these new lines, lifespan and dopaminergic neuronal damage were monitored. 
Pan-neuronal (pUnc-119-driven) expression of AGE-1 is able to rescue lifespan extension conferred by age-1(hx546), while dopaminergic-specific AGE-1 restoration does not impact longevity (Figure 6A-B). These data demonstrate that AGE-1 function is required in neurons in general to regulate lifespan, as previously suggested (Iser et al, 2007; Wolokow et al, 2000), whereas AGE-1 in dopaminergic neurons does not participate in lifespan. At day 14, dopaminergic neuronal damage in LRRK2(G2019S) worms is pronounced (score $=4.1 \pm 0.06$ ), and the introduction of age-1 rescues neuronal damage (score $=2.81 \pm 0.06$ ) (Figure 6C-D). Restoration of AGE-1 expression in dopaminergic neurons (pDat-1::AGE-1) is sufficient to significantly and partially restore neuronal damage induced by LRRK2(G2019S) (score $=3.28 \pm 0.07$ ) (Figure 6CD). These data suggest that interactions between AGE-1 and LRRK2(G2019S) can occur cellautonomously within dopaminergic neurons, despite also having a non-cell-autonomous role in lifespan regulation. Surprisingly, pan-neuronal restoration of AGE-1 further enhances protection (reduces damage) in dopaminergic neurons (score $=2.34 \pm 0.08$ ) beyond the protective effects of age-1 alone (Figure 6C-D). Similar results with respect to lifespan extension and dopaminergic neuronal damage are observed with at least two independent array lines expressing AGE-1 (Figure S3). These complex data suggest that dopaminergic neuronal health may be regulated directly by cell-autonomous AGE-1 signaling, as well as indirectly by non-cell-autonomous inputs arising from outside the nervous system. The observation that expressing AGE-1 in neurons of LRRK2(G2019S); age-1 worms decreases lifespan but increases neuronal survival demonstrates that lifespan and neuroprotection can be experimentally dissociated.

\section{Discussion}

Understanding LRRK2 pathophysiological mechanisms is critical for defining cellular pathways and targets that are amenable to therapeutic intervention for attenuating neurodegeneration in LRRK2-linked PD. In this study, we evaluate how distinct mechanisms regulating the aging process interact with human LRRK2(G2019S)-induced dopaminergic neurodegeneration, addressing whether longevity is intrinsically coupled to dopaminergic neuronal health. We first demonstrate that mean lifespan of LRRK2(G2019S) worms can be successfully extended by different age-related pathways, including reduction of insulin/IGF-1 signaling (daf-2; age-1), TOR signaling (rsks-1), caloric intake (eat-2), chemoperception (osm-5), germline function (glp-1) or mitochondrial function (nuo-6). Next, we compared how LRRK2(G2019S)-induced dopaminergic degeneration was impacted by genetic mutants with different levels of lifespan extension. We find that age-dependent neuronal damage induced by LRRK2(G2019S) is broadly regulated by distinct lifespan-extending pathways, with extended longevity generally correlating with 
dopaminergic neuroprotection. Importantly, our data demonstrate that neuroprotection against LRRK2(G2019S) is not simply limited or specific to daf-2, as we previously reported (Cooper et al, 2015), but extends beyond insulin-IGF-1 signaling. However, we find that lifespan-extending paradigms variably influence the extent of neuroprotection with a non-linear relationship between lifespan and dopaminergic damage in the LRRK2(G2019S) model. These data suggest that lifespan extension per se is not sufficient alone for protection, and supports instead a specific interaction between certain age-related pathways and LRRK2(G2019S)-related pathogenic mechanisms, including most prominently insulin/IGF-1 signaling, TOR-mediated protein translation, and mitochondrial respiration. Our study highlights these age-related pathways as important for LRRK2-linked disease that warrant further mechanistic studies in mammalian LRRK2 models.

The tissue specificity mediating the interaction between LRRK2(G2019S) and lifespanregulating factors was examined using neuronal-specific versus body-wide (non-neuronal) RNAimediated gene silencing. Neuronal-specific or body-wide knockdown of age-1, daf-2, rsks-1, and nuo-6 are sufficient to induce lifespan extension and reduce LRRK2(G2019S)-induced dopaminergic damage, suggesting roles for these pathways in both neuronal and peripheral nonneuronal cells. However, one clear example of lifespan extension being experimentally dissociated from neuroprotection is provided by ife-2 knockdown that extends lifespan with bodywide or neuronal-specific RNAi yet fails to impact dopaminergic degeneration. Interestingly, we find that knockdown of nuo- 6 that induces lifespan extension via mitochondrial dysfunction (Yang et al, 2010), interacts with LRRK2(G2019S), as both a systemic genetic mutant and neuronalspecific RNAi lead to a modest increase in lifespan yet a dramatic reduction of LRRK2(G2019S)induced neurotoxicity. Nuo-6 therefore provides another example of this dissociation that may imply this mitochondrial-related pathway rather than lifespan extension most likely drives the protective interaction with LRRK2(G2019S). The loss of nuo-6 function, and impaired mitochondrial complex-I activity, appears to contradict long-held observations that mitochondrial impairment contributes to PD (Singh et al, 2019). It is important to note that nuo-6 genetic mutation or body-wide RNAi both induce early lethality in worms, but not when limited to neurons, implying that the beneficial effects of lowering nuo- 6 arise solely from neurons. Mitochondrial dysfunction is sensed and adapted to early in C.elegans development (Dillin et al, 2002), leading to upregulation of stress response and other related pathways that may specifically protect against LRRK2(G2019S) later in life. It is most likely therefore that these stress-related adaptations in neurons rather than complex-I deficits induced by reduced nuo- 6 levels are sufficient to mediate neuroprotection. 
Our study also focused on the insulin/IGF-1 signaling pathway as a mechanism of delayed aging and increased neuroprotection in the LRRK2(G2019S) model of PD. Reducing insulin receptor signaling or increasing DAF-16/FOXO activity specifically in adipose or neuroendocrine tissue has been shown to increase lifespan in worms, flies and mice (Apfeld and Fontana, 2018), and has been shown to have both cell-autonomous and non-cell-autonomous roles in the regulation of lifespan and other metabolic processes. In agreement with previous studies (Tank et al, 2011; Toth et al, 2010; Pan et al, 2011; Wolkow et al, 2000; Iser et al, 2007), we find that the insulin/IGF-1 signaling pathway is involved in nervous system aging, including preservation of dopaminergic neuronal integrity and survival. We find that specifically restoring AGE-1/PI3K function in dopaminergic neurons is sufficient to partially reverse the neuroprotective effects against LRRK2(G2019S) mediated by age-1 disruption. However, pan-neuronal restoration of AGE-1 activity, which is capable of reversing lifespan extension in systemic age-1 mutants, appears to have an opposite effect on dopaminergic neurodegeneration induced by LRRK2(G2019S). This unexpected finding demonstrates that the insulin/IGF-1 signaling pathway likely acts in both a cell-autonomous (i.e. dopaminergic) and non-cell-autonomous manner with respect to neuronal health, perhaps through the activation of stress response pathways in nondopaminergic neurons and/or peripheral tissues that elicit further neuroprotection against LRRK2(G2019S). This observation highlights the importance of studying neurodegeneration and potential therapeutic interventions in the context of the whole organism.

\section{Conclusions:}

Our study provides insight into the pathogenesis of LRRK2-linked PD and longevity regulation. We find that LRRK2(G2019S)-induced neurodegeneration is strongly influenced by distinct pathways governing the overall rate of aging and lifespan, including insulin/IGF-1 signaling, the TOR pathway, and mitochondrial respiration. These and other lifespan-regulating pathways are highly conserved and associate with major signaling pathways. While distinct sets of factors are engaged downstream, mechanisms of lifespan modulation at least partially overlap, and crosstalk between signaling pathways may partly account for the observed complexity. LRRK2 has been shown to interact with different cell signaling pathways, specifically those involved in protein degradation, mitochondrial and endolysosomal functions. Further examination of the interplay between these pathways may reveal common factors influencing LRRK2-dependent neurotoxicity, and future studies will require validation of these mechanisms in genetic rodent models of PD. Overall, our study highlights that systemic aging of the organism and neuronal degeneration are related phenomena, but in a non-linear manner, and suggests that insulin/IGF- 
1 signaling is compartmentalized within different cells and tissues of an organism that are potentially differentially regulated during aging. Taken together, our study highlights specific lifespan-regulating pathways that may provide a number of promising therapeutic targets for inhibiting LRRK2-dependent neurodegeneration in PD.

\section{Methods}

\section{C.elegans strains and maintenance:}

Unless noted, strains were maintained at $20^{\circ} \mathrm{C}$ on NGM seeded with OP50 bacteria according to standard practice (Brenner, 1974). Transgenic cwrls856 [Pdat-1::GFP, Pdat-1::LRRK2(G2019S), lin-15(+)] has been previously described (Yao et al, 2010; Cooper et al, 2015). Double mutants were constructed as detailed elsewhere (Cooper et al, 2015). daf-2 animals were raised at $15^{\circ} \mathrm{C}$ and shifted to $20^{\circ} \mathrm{C}$ at L4 stage. glp-1 animals were raised at $15^{\circ} \mathrm{C}$ and shifted to $25^{\circ} \mathrm{C}$ as embryos to obtain germline(-/-) adults. cwrls856 [pDat-1::GFP; pDat-1::LRRK2(G2019S)] were treated similarly in all conditions to serve as controls. For all assays, a minimum of three biological replicates ( 40 worms) per strain were assayed. All assays were performed by an experimenter blinded to genotype.

\section{Lifespan analysis:}

Worm populations were synchronized by washing a well-seeded plate to remove adults and larvae, leaving a population of embryos. After 2-3 days, upon reaching young adulthood, 30-40 worms were transferred to NGM plates containing $25 \mu \mathrm{M}$ fluorodeoxyuridine (FUdR); inclusion of this DNA synthesis inhibitor prevents hatching of progeny following the initial transfer (at $48 \mathrm{~h}$ post-plating). FUdR has minimal effects on longevity at this concentration (Van Raamsdonk \& Hekimi, 2011), and does not affect expression levels or localization of GFP transgenes.

Animals were transferred to fresh plates weekly, and viability was scored every 2 days by gentle prodding with a platinum pick. Animals that failed to respond were scored as dead. Worms that died from internal hatching of progeny, expulsion of internal contents or desiccation on the side of the dish were censored and excluded from analysis.

\section{Dopaminergic neuronal damage:}

Dopaminergic neurons were marked by GFP expression driven by the dopamine transporter (DAT-1) promoter. To quantify damage and compare across treatments, we developed a scoring metric of $0-5$, with a score of 0 given to healthy, unbroken, bright, thick axons and cell bodies lacking punctate structures, as these are not observed in pDat-1::GFP-expressing strains that 
lack the LRRK2(G2019S) transgene. A score of 5 was given to neurons severely damaged (multiple puncta and/or cell body morphology defects) or undetectable GFP. Transmission electron microscopy has been used previously to demonstrate that loss of GFP in these neurons correlates with ultrastructural damage consistent with apoptotic cell death by (Nass et al, 2002).

Quantitative analyses of dopaminergic degeneration was performed by immobilizing 20-40 worms (unless stated otherwise, aged to Day 14 on $25 \mu \mathrm{M} F U d R$ ) on a 1.5\% agarose pad with 5 $\mathrm{mM}$ levamisole. Live worms on coverslipped slides were immediately scored at 40x using a Leica upright epifluorescent microscope (DM5500 B). Unless noted otherwise, all images are maximum projection composite micrographs collected on a Nikon A1plus-RSi scanning confocal microscope. Images were collected every $0.5 \mu \mathrm{m}$ over $30 \mu \mathrm{m}$, capturing the cell body and axonal projections of pDat-1::GFP-labeled cephalic sensilla (CEP) neurons. All images shown are representative of multiple independent experiments.

\section{RNAi}

Standard NGM agar was supplemented with $50 \mu \mathrm{g} / \mathrm{ml}$ carbenicillin and $5 \mathrm{mM}$ isopropyl $\beta$-D-1thiogalactopyranoside (IPTG) post-autoclaving and allowed to dry overnight at room temperature. RNAi cultures were prepared by sequence verifying single colony cultures from the Ahringer RNAi plasmid library. E.coli HT115 carrying sequence-verified RNAi targeting plasmid clones were grown in LB supplemented with $50 \mu \mathrm{g} / \mathrm{ml}$ carbenicillin and grown for 8 hours at $37^{\circ} \mathrm{C}$. Cultures were concentrated $5 x$ prior to seeding plates with $200 \mu$ of RNAi bacteria, which was spread, dried, and allowed to induce dsRNA expression for 48 hours at RT, after which point plates were stored at $4^{\circ} \mathrm{C}$ for up to 1 week. HT115 carrying the pL4440 plasmid lacking a gene-targeting cassette was used as a control for all RNAi experiments (empty vector).

RNAi knockdown was initiated by plating L4 $\mathrm{P}_{\mathrm{o}}$ animals to prepared plates. After 30-35 h, adult animals were transferred to fresh RNAi plates and allowed to lay for $12 \mathrm{~h}$, after which point adults were removed. After an additional $\sim 50 \mathrm{~h}$, when embryos laid on RNAi plates had developed to young adulthood, animals were transferred to fresh RNAi plates containing $25 \mu \mathrm{M}(\mathrm{FUdR})$ in addition to carbenicillin and IPTG.

Neuron-specific RNAi was performed by crossing TU3401 (sid-1(pk3321); uls69[pCFJ90(myo-2p::mCherry) + unc-119p::sid-1]) (Calixto et al, 2010) to cwrls856 [pDat1::GFP; pDat-1::LRRK2(G2019S)]. Lifespan and neuronal damage were assayed as above, however, due to the modestly shortened lifespan attributed to sid-1(pk3321) in the periphery, dopaminergic neuronal damage was assayed at Day 11, a point at which control animals show equivalent damage to non-sid-1 animals at Day 14. 


\section{Construction of AGE-1 transgenics}

Tissue-specific rescue transgenic expression constructs of the AGE-1 ORF were generated by standard Multi-site Gateway cloning methods. Full-length age-1 cDNA was PCR-amplified using Phusion High-Fidelity DNA Polymerase+GC buffer (Thermofisher) using total cDNA prepared from N2 mRNA (Ampliscribe). AttB1 and AttB2 adaptors were added to the 5' end of the forward and reverse primers, respectively, to allow for BP-mediated recombination into a pDONR221 vector (Invitrogen). The resulting entry clone was sequence verified. LR recombination (Invitrogen) was used to generate AGE-1 flanked by a 5' promoter element (either pan-neuronal pUnc-119 or dopaminergic-specific pDat-1, both from Dharmacon), and a 3'UTR (pADA-126; let-858 3'UTR in pDONRP2R-P3) in the pCFJ150 MosSCl destination vector. Resulting expression plasmids were confirmed by diagnostic digest. Injection mixes were prepared by digesting pMS16 (pUnc-119::AGE-1::let-858) and pMS17 (pDat-1::AGE-1::let-858) with Agel and Srf1; the resulting $\sim 11.6 \mathrm{~kb}$ band was gel purified and injected into N2 animals at 5 $\mathrm{ng} / \mu \mathrm{l}$ with co-marker (pMyo-3::RFP (bsem1122) at $5 \mathrm{ng}$ ) and carrier DNA (sheared salmon testis DNA) to $100 \mathrm{ng} / \mu \mathrm{l}$. The presence of the AGE-1 ORF transgene was verified in RFP positive lines by PCR. AGE-1/RFP double-positive arrays were then crossed to age-1(hx546); cwrls856 [pDat1::GFP; pDat-1::LRRK2(G2019S)] to generate tissue-specific restoration lines. Homozygosity of the $h \times 546$ missense mutant was confirmed using intron-specific primers, allowing for genomic locus-specific sequencing.

\section{Statistical analysis:}

All statistical significance was determined using GraphPad Prism. Lifespan assays were assessed using the log-rank test to compare survival curves. Statistical significance of differences of lifespan means and dopaminergic neuronal damage was determined using a one-way ANOVA with Bonferroni or Tukey post-hoc tests to identify specific differences. Data are presented as mean \pm standard error of the mean (SEM).

\section{C.elegans strains used in this study:}

\begin{tabular}{llc} 
Strain & Genotype & Original publication \\
\hline SGC730 & cwrls730 [Pdat-1::GFP, lin-15(+)] & Yao et al, 2010 \\
SGC722 & cwrls722 [Pdat-1::GFP, Pdat-1::LRRK2(WT), lin-15(+)] & Yao et al, 2010; Cooper et al, 2015 \\
CB1370 & daf-2(e1370) III & Kenyon et al, 1993; Kimura et al, 1997
\end{tabular}




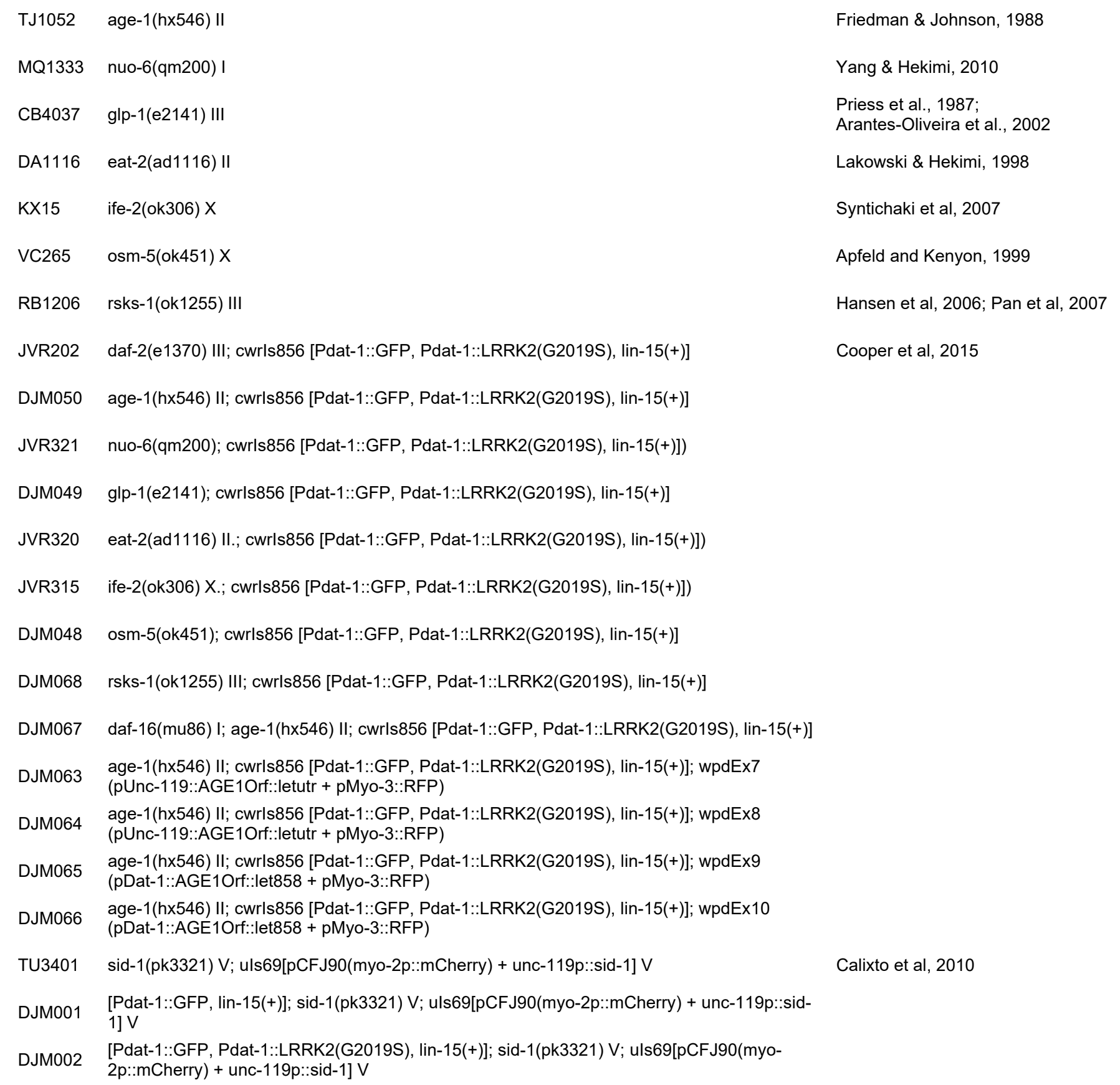

\section{Acknowledgments:}

This work was supported by funding from the National Institutes of Health (R21AG058241) and Van Andel Institute. We thank Dr. Shu Chen (Case Western Reserve University) for providing the human LRRK2 transgenic strain. Some strains were provided by the CGC, which is funded by NIH Office of Research Infrastructure Programs (P40OD010440). We thank Dr. Jason Cooper (VAI) and Jennifer Kordich (VAI) for technical assistance in generating some worm strains. 


\section{References:}

Apfeld J, Fontana W. Age-Dependence and Aging-Dependence: Neuronal Loss and Lifespan in a C. elegans Model of Parkinson's Disease. Biology 2017 Dec 23;7(1)

Apfeld J, Kenyon C. Regulation of lifespan by sensory perception in Caenorhabditis elegans. Nature 1999; 402(6763):804-809

Apfeld J, Kenyon C. Cell nonautonomy of C. elegans daf-2 function in the regulation of diapause and life span. Cell 95, 199-210 (1998)

Arantes-Oliveira N, Apfeld J, Dillin A, Kenyon C. Regulation of Life-Span by Germ-Line Stem Cells in Caenorhabditis elegans. Science 2002; Vol. 295, Issue 5554, pp. 502-505

Asikainen S, Vartiainen S, Lakso M, Nass R, Wong G. Selective sensitivity of Caenorhabditis elegans neurons to RNA interference. Neuroreport 2005; 16: 1995-1999.

Bettinger JC McIntire SL. State-dependency in C. elegans. Genes, Brain and Behavior 2004; 3(5):266-72.

Berman JR, Kenyon C. Germ-cell loss extends $C$. elegans life span through regulation of DAF16 by kri-1 and lipophilic-hormone signaling. Cell 2006; 124(5):1055-68.

Brenner S. The genetics of Caenorhabditis elegans. Genetics. 1974; 77(1):71-94.

Billingsley KJ, Bandres-Ciga S, Saez-Atienzar S, Singleton AB. Genetic risk factors in Parkinson's disease. Cell Tissue Research. 2018 Jul;373(1):9-20.

Calixto A, Chelur D, Topalidou I, Chen X, Chalfie M. Enhanced neuronal RNAi in C. elegans using SID-1. Nature Methods 2010; 7(7): 554-559

Cao S, Gelwix CC, Caldwell KA, Caldwell GA. Torsin-Mediated Protection from Cellular Stress in the Dopaminergic Neurons of Caenorhabditis elegans. Journal of Neuroscience 2005; 25(15): 3801-3812

Collier TJ, Kanaan NM, Kordower JH. Aging and Parkinson's disease: Different sides of the same coin? Mov Disord. 2017, 32(7):983-990

Cooper JF, Dues DJ, Spielbauer KK, Machiela E, Senchuk MM, Van Raamsdonk JM. 2015. Delaying aging is neuroprotective in Parkinson's disease: a genetic analysis in $C$. elegans models. NPJ Parkinsons Dis. 2015; 1:15022

Chew YL, Fan X, Götz J, Nicholas, HR. Aging in the nervous system of Caenorhabditis elegans. Commun Integr Biol. 2013; 6(5): e25288.

de Lau LM, Breteler MM. Epidemiology of Parkinson's disease. Lancet Neurology 2006; $5(6): 525-35$.

Dillin A, Hsu AL, Arantes-Oliveira N, Lehrer-Graiwer J, Hsin H, Fraser AG, Kamath RS, Ahringer $\mathrm{J}$, Kenyon C. Rates of behavior and aging specified by mitochondrial function during development. Science 2002;298(5602):2398-401. 
Dorsey ER, Sherer T, Okun MS, Bloem BR. The emerging evidence of the Parkinson pandemic. Journal of Parkinsons Disease 2018; 8(s1):S3-S8.

Duerr JS, Frisby DL, Gaskin J, Duke A, Asermely K, Huddleston D, Eiden LE, Rand JB. The cat1 gene of Caenorhabditis elegans encodes a vesicular monoamine transporter required for specific monoamine-dependent behaviors. Journal of Neuroscience. 1999 Jan 1;19(1):72-84.

Durieux J, Wolff S, Dillin A. The cell-non-autonomous nature of electron transport chain-mediated longevity. Cell 2011;144(1):79-91.

Feng J, Bussiere F, Hekimi S. Mitochondrial electron transport is a key determinant of life span in Caenorhabditis elegans. Developmental Cell. 2001;1(5):633-44

Friedman DB, Johnson TE. A mutation in the age-1 gene in Caenorhabditis elegans lengthens life and reduces hermaphrodite fertility. Genetics 1998; 118(1):75-86.

Fujii M, Matsumoto Y, Tanaka N, Miki K, Suzuki T, Ishii N, Ayusawa D. Mutations in Chemosensory Cilia Cause Resistance to Paraquat in Nematode Caenorhabditis elegans. Journal of Biological Chemistry 2004, 7;279(19):20277-82

GBD 2016 Neurology Collaborators. Global, regional, and national burden of neurological disorders, 1990-2016: a systematic analysis for the Global Burden of Disease Study 2016. Lancet Neurology 2019 May;18(5):459-480.

Hamamichi S, Rivas RN, Knight AL, Cao S, Caldwell KA, Caldwell GA. Hypothesis-based RNAi screening identifies neuroprotective genes in a Parkinson's disease model. Proc Natl Acad Sci 2008, 15; 105(2): 728-733.

Hansen M, Taubert S, Crawford D, Libina N, Lee SJ, Kenyon C. Lifespan extension by conditions that inhibit translation in Caenorhabditis elegans. Aging Cell 2007; 6(1):95-110.

Haugarvoll K, Wszolek ZK. Clinical features of LRRK2 parkinsonism. Parkinsonism Related Disorders. 2009 Dec;15 Suppl 3:S205-8.

Hernandez DG, Reed X, Singleton AB. Genetics in Parkinson disease: Mendelian versus nonMendelian inheritance. Journal of Neurochemistry. 2016 Oct;139 Suppl 1:59-74.

Herndon LA, Schmeissner PJ, Dudaronek JM, Brown PA, Listner KM, Sakano Y, Paupard MC, Hall DH, Driscoll M. Stochastic and genetic factors influence tissue-specific decline in ageing $C$. elegans. Nature 2002; 419(6909):808-14.

Hills T, Brockie PJ, Maricq AV. Dopamine and Glutamate Control Area-Restricted Search Behavior in Caenorhabditis elegans. Journal of Neuroscience 2004, 24 (5) 1217-1225

Ho PW, Leung CT, Liu H, Pang SY, Lam CS, Xian J, Li L, Kung MH Ramsden DB, Ho SL. Agedependent accumulation of oligomeric SNCA/a-synuclein from impaired degradation in mutant LRRK2 knockin mouse model of Parkinson disease: role for therapeutic activation of chaperone-mediated autophagy (CMA). Autophagy 2020; 16(2): 347-370. 
Iser, W. B. \& Wolkow, C. A. DAF-2/insulin-like signaling in C. elegans modifies effects of dietary restriction and nutrient stress on aging, stress and growth. PLOS ONE 2007; 2, e1240.

Jose AM, Smith JJ, Hunter CP (2009) Export of RNA silencing from C. elegans tissues does not require the RNA channel SID-1. Proc Natl Acad Sci 2009; 106: 2283-2288

Kamath RS, Martinez-Campos M, Zipperlen P, Fraser AG, Ahringer J. Effectiveness of specific RNA-mediated interference through ingested double-stranded RNA in Caenorhabditis elegans. Genome Biology 2001;2(1)

Kamath RS, Ahringer J. Genome-wide RNAi screening in Caenorhabditis elegans. Methods 2003; 30(4):313-21.

Kenyon C. A pathway that links reproductive status to lifespan in Caenorhabditis elegans. Ann N Y Acad Sci. 2010; 1204():156-62.

Kenyon C, Chang J, Gensch E, Rudner A, Tabtiang R. A C. elegans mutant that lives twice as long as wild type. Nature 1993; 366, pp. 461-464

Kim J, Daadi MM. Non-cell autonomous mechanism of Parkinson's disease pathology caused by G2019S LRRK2 mutation in Ashkenazi Jewish patient: Single cell analysis. Brain Research 2019; 1722:146342

Kim JK, Gabel HW, Kamath RS, Tewari M, Pasquinelli A, Rual JF, Kennedy S, Dybbs M, Bertin N, Kaplan JM, Vidal M, Ruvkun G. Functional Genomic Analysis of RNA Interference in C. elegans. Science 2005; 308: 1164-1167

Kimura KD, Tissenbaum HA, Y. Liu, G. Ruvkun. daf-2, an insulin receptor-like gene that regulates longevity and diapause in Caenorhabditis elegans. Science 1997; 277 pp. 942-946

Lakowski B, Hekimi S. The genetics of caloric restriction in Caenorhabditis elegans. Proc Natl Acad Sci 1998; 95(22): 13091-13096.

Lapierre LR, Hansen M. Lessons from C. elegans: Signaling pathways for longevity. Cell 2006; 124(5):1055-68.

Lee SS, Lee RY, Fraser AG, Kamath RS, Ahringer J, Ruvkun G. A systematic RNAi screen identifies a critical role for mitochondria in C. elegans longevity. Nature Genetics. 2003;33(1):408.

Libina, N., Berman, J. R. \& Kenyon, C. Tissue-specific activities of C. elegans DAF-16 in the regulation of lifespan. Cell 115, 489-502 (2003).

Liu Z, Hamamichi S, Lee DB, Yang D, Ray A, Caldwell GA, Caldwell KA, Dawson TM, Wanli W. Smith WW, Valina L. Dawson. Inhibitors of LRRK2 kinase attenuate neurodegeneration and Parkinson-like phenotypes in Caenorhabditis elegans and Drosophila Parkinson's disease models. Human Molecular Genetics. 2011 Oct 15; 20(20): 3933-3942.

Loer CM and Kenyon CJ. Serotonin-deficient Mutants and Male Mating Behavior in the Nematode Caenorhabditis elegans. Journal of Neuroscience 1993, 13 (12): 5407-5417. 
Longo F, Mercatelli D, Novello S, Arcuri L, Brugnoli A, Vincenzi F, Russo I, Berti G, Mabrouk OS, Kennedy RT, Shimshek DR, Varani K, Bubacco L, Greggio E, Morari M. Age-dependent dopamine transporter dysfunction and Serine129 phospho- $\alpha$-synuclein overload in G2019S LRRK2 mice. Acta Neuropathol Commun. 2017; 5(1):22.

Marder K, Wang Y, Alcalay RN, Mejia-Santana H, Tang MX, Lee A, et al. Age-specific penetrance of LRRK2 G2019S in the Michael J. fox Ashkenazi Jewish LRRK2 consortium. Neurology 2015; 85:89-95.

Nass R, Hall DH, Miller DM, Blakely RD. Neurotoxin-induced degeneration of dopamine neurons in Caenorhabditis elegans. PNAS 2002; 99 (5) 3264-3269

Nass R and Blakely R. The Caenorhabditis elegans Dopaminergic System: Opportunities for Insights into Dopamine Transport and Neurodegeneration. Annual Review of Pharmacology and Toxicology 2003 43:1, 521-544

Pan CL, Peng CY, Chen CH, Mclntire S. Genetic analysis of age-dependent defects of the Caenorhabditis elegans touch receptor neurons. Proc Natl Acad Sci 2011;108:9274-9.

Pan KZ, Palter JE, Rogers AN, Olsen A, Chen A, Lithgow GJ, Kapahi P. Inhibition of mRNA translation extends lifespan in Caenorhabditis elegans. Aging Cell 2007; 6(1):111-119

Pang SY, Ho PW, Liu HF, Leung CT, Li L, Eun E, Chang S, Ramsden DB, Ho SL. The interplay of aging, genetics and environmental factors in the pathogenesis of Parkinson's disease. Translational Neurodegeneration. 2019; 8: 23.

Priess JR, Schnabel H, Schnabel R. The glp-1 locus and cellular interactions in early C. elegans embryos. Cell 1987; (51)601-611

Sanyal S, Wintle RF, Kindt KS, Nuttley WM, Arvan R, Fitzmaurice P, Bigras E, Merz DC, Hébert TE, van der Kooy D, Schafer WR, Culotti JG, Van Tol HH. Dopamine modulates the plasticity of mechanosensory responses in Caenorhabditis elegans. EMBO J. 2004 28;23(2):473-82.

Sawin, E.R., Ranganathan, R. and Horvitz, H.R. C. elegans locomotory rate is modulated by the environment through a dopaminergic pathway and by experience through a serotonergic pathway. Neuron 2000; 26: 619-631.

Singh A, Zhi L, Zhang H. LRRK2 and mitochondria: Recent advances and current views. Brain Research 2019, 1;1702:96-104

Spatola M and Wider C. Genetics of Parkinson's disease: the yield. Parkinsonism Related Disorders 2014; 20 Suppl 1:S35-8.

Sulston J, Dew M, Brenner S. Dopaminergic neurons in the nematode Caenorhabditis elegans. Journal of Comparative Neurology 1975; 163 (2): 215-226

Syntichaki P, Troulinaki K, Tavernarakis N. elF4E function in somatic cells modulates ageing in Caenorhabditis elegans. Nature 2007; 445, 922-926

Tank EM, Rodgers KE, Kenyon C. Spontaneous age-related neurite branching in Caenorhabditis elegans. Journal of Neuroscience 2011; 31:9279-88. 
Timmons L, Court DL, Fire A (2001) Ingestion of bacterially expressed dsRNAs can produce specific and potent genetic interference in Caenorhabditis elegans. Gene 2001; 263: 103-112

Tissenbaum HA. DAF-16: FOXO in the Context of C. elegans. Current Topics in Developmental Biology 2018; 127:1-21.

Toth ML, Melentijevic I, Shah L, Bhatia A, Lu K, Talwar A, Naji H, Ibanez-Ventoso C, Ghose $P$, Jevince A, Xue J, Herndon LA, Bhanot G, Rongo C, Hall DH, Driscoll M. Neurite sprouting and synapse deterioration in the aging Caenorhabditis elegans nervous system. Journal of Neuroscience 2012; 32:8778-90.

Tucci M.L., Harrington A.J., Caldwell G.A., Caldwell K.A. (2011) Modeling Dopamine Neuron Degeneration in Caenorhabditis elegans. In: Manfredi G., Kawamata H. (eds) Neurodegeneration. Methods in Molecular Biology (Methods and Protocols), vol 793. Humana Press, Totowa, NJ

Van Raamsdonk JM, Hekimi S. FUdR causes a twofold increase in the lifespan of the mitochondrial mutant gas-1. Mech Ageing Dev. 2011; 132: 519-521.

Wang D, Kennedy S, Conte D Jr, Kim JK, Gabel HW, Kamath RS, Mello CC, Ruvkun G. Somatic misexpression of germline $P$ granules and enhanced RNA interference in retinoblastoma pathway mutants. Nature 2005; 436: 593-597

Wolkow, C. A., Kimura, K. D., Lee, M. S. \& Ruvkun, G. Regulation of C. elegans life-span by insulinlike signaling in the nervous system. Science 2000; 290, 147-150.

Xiong Y, Neifert S, Karuppagounder SS, Stankowski JN, Lee BD, Grima JC, Chen G, Ko HS, Lee Y, Swing D, Tessarollo L, Dawson TM, Dawson VL. Overexpression of Parkinson's DiseaseAssociated Mutation LRRK2 G2019S in Mouse Forebrain Induces Behavioral Deficits and $\alpha-$ Synuclein Pathology. eNeuro. 2017; 4(2)

Yao C, El Khoury R, Wang W, Byrd TA, Pehek EA, Thacker C, Zhu X, Smith MA, Wilson-Delfosse AL, Chen SG. LRRK2-mediated neurodegeneration and dysfunction of dopaminergic neurons in a Caenorhabditis elegans model of Parkinson's disease. Neurobiology of Disease. 2010; 40(1):73-81

Yang W, Hekimi S. Two modes of mitochondrial dysfunction lead independently to lifespan extension in Caenorhabditis elegans. Aging Cell 2010 Jun;9(3):433-47

Yin JA, Liu XJ, Yuan J, Jiang J, Cai SQ. Longevity Manipulations Differentially Affect Serotonin/Dopamine Level and Behavioral Deterioration in Aging Caenorhabditis elegans. Journal of Neuroscience 2014; 34 (11) 3947-3958

Zhang, P., Judy, M., Lee, S. J. \& Kenyon, C. Direct and indirect gene regulation by a life-extending FOXO protein in C. elegans: roles for GATA factors and lipid gene regulators. Cell Metabolism, 2013 17, 85-100 
Figure legends:

Figure 1. Human LRRK2(G2019S) expression in worms induces age-dependent dopaminergic neuronal damage without altering lifespan. A) Dopaminergic neurons are marked by GFP driven by the dopamine transporter (Dat-1) promoter. The pDat1::hLRRK2(G2019S); pDat-1::GFP line (cwrls856) is referred to as LRRK2(G2019S). Representative maximum projection confocal micrographs from LRRK2(G2019S) transgenic worms indicating increased GFP-positive dopaminergic neuronal damage during aging. Images are oriented with the mouth to the left. B) C.elegans dopaminergic signaling is carried out by 8 neurons from a total of 302 neurons. Two pairs of cephalic neurons (CEP, blue arrows) are the most anterior, followed by the anterior deirids (ADE, red arrows) located proximal to the nerve ring, and the laterally positioned posterior deirid pair (PDE). The axonal processes of the CEP neurons (red asterisk) extend toward the anterior, while the branched neuritic processes project distally into the synapse-rich nerve ring. The 0-5 scoring metric accounts for the overall morphology and damage of individual dopaminergic cell soma and neurites, the presence and localization of GFP-positive dense puncta (internal vs. external to cell body), and overall GFP intensity within individual neurons. C) Graph comparing dopaminergic neuronal damage to CEP neurons in worms expressing LRRK2(G2019S) or GFP alone at days 9, 12 and 15. Data represent dopaminergic damage with scoring from 0-5 (mean \pm SEM; $n \geq 3$ independent experiments). ${ }^{\#} P<0.05$ or ${ }^{\# \#} P<0.0001$ by one-way ANOVA with Tukey's post-hoc test as indicated, or ${ }^{* * * *} P<0.0001$ by unpaired Student's $t$-test comparing GFP to LRRK2(G2019S) at each time point. LRRK2(G2019S) induces marked and progressive neuronal damage with age compared to GFP alone. D-E) LRRK2(G2019S) or GFP lines with transgene expression restricted to dopaminergic neurons (Pdat-1) exhibit a normal lifespan compared to wild-type N2 control worms as indicated by Kaplan-Meier survival analysis (D) and mean lifespan (E, mean \pm SEM, $n=3$ experiments, with 30 animals per experiment). E) One-way ANOVA finds no significant differences between mean lifespans of wild-type (N2, black), cwrls730 [pDat-1::GFP] (green), and cwrls856 [pDat1::GFP; pDat-1::LRRK2(G2019S)] (blue) worm strains.

Figure 2. Genetic mutation of negative longevity regulators extends the lifespan of LRRK2(G2019S) transgenic worms. A-H) Kaplan-Meier survival curves comparing longevity of wild-type (N2, black solid) and LRRK2(G2019S) (black dashed) worms with lifespan-extending genetic mutants alone (color solid) and LRRK2(G2019S) transgenics on each mutant background (color dashed). Curves represent combined data from at least 3 individual experiments $(n=30$ 
animals per experiment). I) Mean lifespan from data shown in A-H. Bars represent mean \pm SEM.

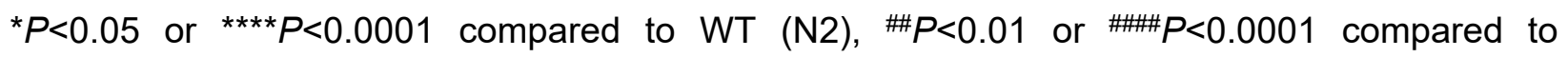
LRRK2(G2019S), by one-way ANOVA with Bonferroni's multiple comparisons test.

Figure 3. Lifespan extension is broadly neuroprotective in the LRRK2(G2019S) worm model. A) Representative maximum projection confocal images of anterior GFP-positive CEP dopaminergic neurons at day 14 of adulthood from LRRK2(G2019S) on a wild-type or genetic mutant background. Images reveal reduced yet variable neuronal damage in all lifespanextending backgrounds. B) Quantification of CEP dopaminergic neuronal damage at day 14 using the $0-5$ scoring metric. Bars represent the mean \pm SEM damage from $\geq 3$ individual experiments ( $n=40$ animals per experiment). ${ }^{*} P<0.05$, ${ }^{* *} P<0.005$ or ${ }^{* * * *} P<0.0001$ compared to LRRK2(G2019S) alone by one-way ANOVA with Dunnett's post-hoc test. C) Linear regression analysis $\left(R^{2}=0.4266\right)$ of mean lifespan (in days) relative to mean dopaminergic neuronal damage (0-5 score) for each worm strain, reveals a general inverse correlation indicating that lifespan extension is broadly associated with reduced neuronal damage.

Figure 4. Lifespan extension and neuroprotection mediated by age-1 mutant in LRRK2(G2019S) worms is dependent on DAF-16. Kaplan-Meier survival curve (A) and mean lifespan (B) of LRRK2(G2019S) worms on a wild-type background, and on an age-1 or age-1; daf-16 genetic background. The daf-16 mutant fully reverses the extended lifespan conferred by age-1. A) Curves represent combined data from at least 3 individual experiments $(n=30$ animals per experiment), whereas B) bars represent mean \pm SEM. C) Quantitation of CEP dopaminergic neuronal damage at day 14 in LRRK2(G2019S) worms on all three genetic backgrounds. An age1 mutant markedly reduces neuronal damage compared to the wild-type background, that is fully restored to normal wild-type levels in an age-1; daf-16 double mutant. Bars represent the mean \pm SEM neuronal damage ( $n \geq 3$ independent experiments; with 40 animals/experiment). ${ }^{* * * *} P<0.0001$ compared to LRRK2(G2019S) alone by one-way ANOVA with Dunnett's post-hoc analysis.

Figure 5. Neuronal-specific knockdown of multiple longevity-regulating genes is neuroprotective in the LRRK2(G2019S) worm model. A subset of negative regulators of longevity were targeted by RNAi-mediated knockdown either systemically (body-wide, with reduced efficacy in neurons) $(\mathbf{A}, \mathbf{C})$ or specifically within the neuronal cell population using the pUnc-119::SID-1; sid-1(pk3321) background (B, D). Mean lifespan of each strain is indicated (A- 
B). Quantitation of CEP dopaminergic neuronal damage in LRRK2(G2019S) worms with C) systemic body-wide RNAi assessed at day 14, or D) neuronal-specific RNAi assessed at day 11. Note: systemic knockdown of nuo-6 results in early lethality and cannot be assessed in (A, C). Bars represent the mean \pm SEM combined from $\geq 3$ independent experiments with $n \geq 30$ animals per experiment. ${ }^{*} P<0.05,{ }^{* *} P<0.01,{ }^{* *} P<0.001$ or ${ }^{* * * *} P<0.0001$ compared to control (empty vector, EV) by one-way ANOVA with Dunnett's post-hoc test.

Figure 6. Restoring AGE-1/PI3K function in dopaminergic neurons demonstrates a cellautonomous effect on LRRK2(G2019S)-induced neuronal damage. A) Kaplan-Meier survival curves comparing the lifespan of LRRK2(G2019S) (black), age-1; LRRK2(G2019S) (blue), and transgenic lines expressing AGE-1 from pan-neuronal (pUnc-119; green) or dopaminergicspecific (pDat-1; red) promoters in the age-1; LRRK2(G2019S) background. B) Mean lifespan or each worm strain from survival data shown $(\mathbf{A})$. Extended lifespan in the age-1 background is partially reverted by pan-neuronal AGE-1 expression. C) Quantification of mean dopaminergic neuronal damage induced by LRRK2(G2019S) at day 14 in wild-type, age-1 mutant, or in tissuespecific AGE-1 rescue lines on an age-1 mutant background. B-C) Bars represent the mean \pm SEM from $\geq 3$ independent experiments with $n \geq 30$ animals per experiment. ${ }^{* * * *} P<0.0001$ between the indicated groups by one-way ANOVA with Dunnett's multiple comparisons post-hoc test. D) Representative maximum projection confocal images of GFP-positive CEP dopaminergic neurons at day 14 in pDat-1::GFP; pDat-1::LRRK2(G2019S) worms on wild-type, age-1 or transgenic $A G E-1$ rescue backgrounds.

\section{Supplemental figure legends:}

Figure S1. Systemic or neuronal-specific RNAi targeting chemosensory perception pathways fail to extend lifespan of LRRK2(G2019S) worms. An RNAi clone targeting osm-5 was not available in the Ahringer RNAi library, therefore, we tested alternative candidates from this pathway for their ability to extend lifespan based on the work of Fujii et al, 2004. RNAi clones targeting che-3 and osm-6 both reveal extremely modest yet non-significant lifespan extension in systemic LRRK2(G2019S) or neuronal-specific LRRK2(G2019S); SID-1 worm strains at $100 \mu \mathrm{M}$ FUdR. Shown are Kaplan-Meier survival curves (A-B) and mean lifespan for C) systemic or D) neuronal-specific che-3 and osm-5 RNAi. C-D) Bars represent mean \pm SEM from $\geq 3$ independent experiments (with $n \geq 30$ animals/experiment). Differences between groups are not significant by one-way ANOVA with Dunnett's post-hoc test. 
Figure S2. Kaplan-Meier survival curves comparing lifespan extension by systemic or neuronal-specific RNAi in LRRK2(G2019S) transgenic worms. Expression in the wild-type background confers systemic body-wide RNAi whereas a sensitized background [pUnc-119::SID1; sid-1(pk3321)] confers neuronal-specific RNAi in LRRK2(G2019S) transgenic worms. Curves represent combined data from $\geq 3$ individual experiments ( $n=30$ animals per experiment). Panneuronal or systemic knockdown of age-1 and ife-2 increase lifespan to similar extents, whereas neuronal-specific RNAi for daf-2 and rsks-1 extends lifespan to a lesser extent than systemic RNAi. Systemic RNAi for nuo-6 is lethal.

Figure S3. Lifespan and dopaminergic neuronal damage in LRRK2(G2019S); age-1 worms with pan-neuronal or dopaminergic-specific expression of AGE-1/PI3K from independent transgenic arrays. A) Kaplan-Meier survival curves comparing the lifespan of LRRK2(G2019S) (black), age-1; LRRK2(G2019S) (blue), and independent transgenic arrays (line 2) with panneuronal (pUnc-119, green) or dopaminergic-specific (pDat-1, red) AGE-1 expression in age-1; LRRK2(G2019S) worms. Curves represent combined data from $\geq 3$ individual experiments $(n=$ 30 animals per experiment). B) Mean lifespan of each worm strain derived from (A). C) Quantification of dopaminergic neuronal damage at day 14 induced by LRRK2(G2019S) on a wild-type or age-1(-) background, or with tissue-specific restoration of AGE-1 on an age-1(-) background. B-C) Bars represent the mean \pm SEM from $\geq 3$ independent experiments with $n \geq 30$ animals/experiment. ${ }^{* *} P<0.005$, ${ }^{* * *} P<0.001$ or ${ }^{* * *} P<0.0001$ between indicated groups by oneway ANOVA with Tukey's multiple comparisons post-hoc test. 
Table 1. Comparison of lifespan extension induced by genetic mutants in wild-type and LRRK2(G2019S) worms

\begin{tabular}{|c|c|c|c|}
\hline Worm strain & $\begin{array}{c}\text { Mean } \\
\text { Lifespan } \\
\text { (days) }\end{array}$ & $\begin{array}{c}\text { Maximum } \\
\text { Lifespan } \\
\text { (days) }\end{array}$ & $\begin{array}{c}\text { Mean Lifespan } \\
\text { (\% of N2 or } \\
\text { G2019S) }\end{array}$ \\
\hline Wild-type (N2) & 16.43 & 31 & - \\
LRRK2(G2019S) & $\mathbf{1 7 . 0 8}$ & $\mathbf{2 9}$ & - \\
daf-2 (e1370) & 39.28 & 60 & 239.07 \\
daf-2; G2019S & 39.06 & $\mathbf{5 7}$ & $\mathbf{2 2 8 . 6 9}$ \\
age-1 (hx546) & 30.81 & 49 & 187.52 \\
age-1; G2019S & $\mathbf{3 3 . 3 6}$ & $\mathbf{5 1}$ & 195.32 \\
nuo-6 (qm200) & 28.7 & 43 & 174.68 \\
nuo-6; G2019S & $\mathbf{2 6 . 5 6}$ & $\mathbf{4 4}$ & 155.50 \\
rsks-1 (ok1255) & 27.3 & 34 & 166.16 \\
rsks-1; G2019S & $\mathbf{2 6 . 0 1}$ & $\mathbf{3 4}$ & $\mathbf{1 5 2 . 2 8}$ \\
eat-2 (ad1116) & 24.65 & 36 & 150.03 \\
eat-2; G2019S & $\mathbf{2 6 . 4 3}$ & $\mathbf{3 8}$ & $\mathbf{1 5 4 . 7 4}$ \\
glp-1 (e2141) & 28.48 & 42 & 173.34 \\
glp-1; G2019S & $\mathbf{2 9 . 5 6}$ & $\mathbf{4 4}$ & $\mathbf{1 7 3 . 0 7}$ \\
ife-2 (ok306) & 23.55 & 35 & 143.34 \\
ife-2; G2019S & $\mathbf{2 4 . 6 3}$ & $\mathbf{4 0}$ & $\mathbf{1 4 4 . 2 0}$ \\
osm-5 (ok451) & 19.42 & 29 & 118.20 \\
osm-5; G2019S & $\mathbf{2 0 . 4 6}$ & $\mathbf{3 3}$ & $\mathbf{1 1 9 . 7 9}$ \\
\hline
\end{tabular}

For \% mean lifespan, single genetic mutants were compared to N2 worms, whereas double mutants were compared to LRRK2(G2019S) (in bold). 
Table 2. Comparison of fold change in lifespan and dopaminergic neuronal damage with systemic or neuronalspecific RNAi knockdown of longevity-regulating genes

\begin{tabular}{|c|c|c|c|c|}
\hline \multirow{2}{*}{ RNAi } & \multicolumn{2}{|c|}{$\begin{array}{c}\text { Lifespan } \\
\text { (fold change vs. EV) }\end{array}$} & \multicolumn{2}{c|}{$\begin{array}{c}\text { Dopaminergic Damage } \\
\text { (fold change vs. EV) }\end{array}$} \\
\cline { 2 - 5 } & Systemic & Neuronal & Systemic & Neuronal \\
\hline daf-2 & 1.23 & 1.16 & 0.88 & 0.9 \\
age-1 & 1.35 & 1.46 & 0.78 & 0.83 \\
rsks-1 & 1.19 & 1.08 & 0.87 & 0.89 \\
ife-2 & 1.2 & 1.2 & 0.95 & 1.02 \\
nuo-6 & $\mathrm{n} / \mathrm{a}$ & 1.01 & $\mathrm{n} / \mathrm{a}$ & 0.73 \\
\hline
\end{tabular}




\section{A Day 7}
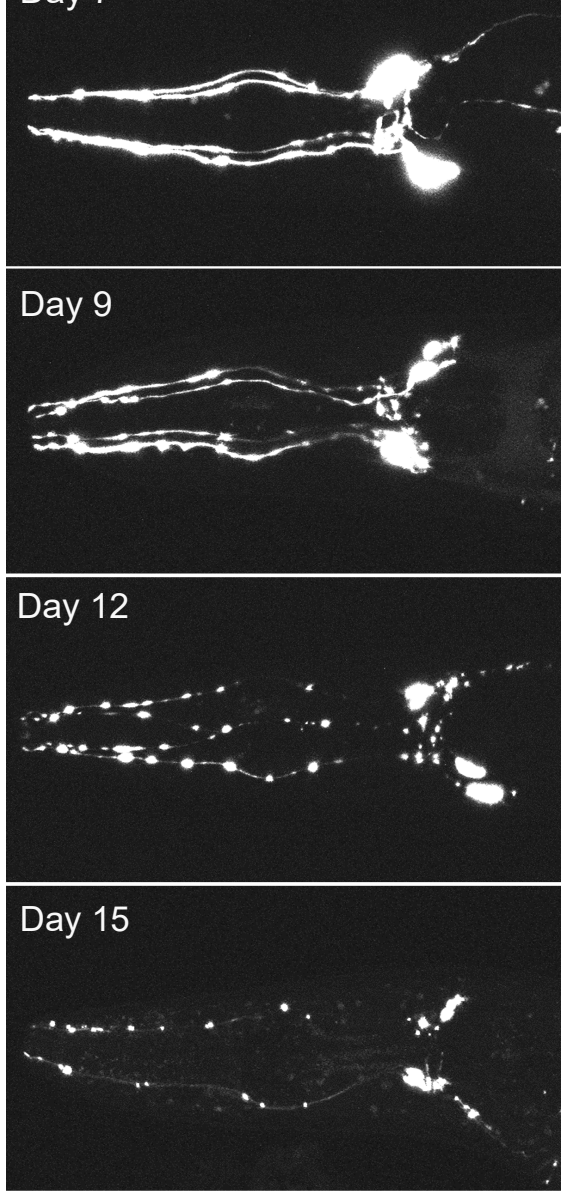

B

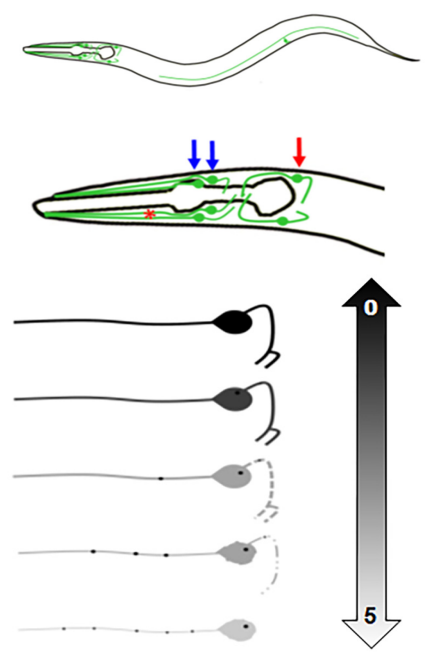

D

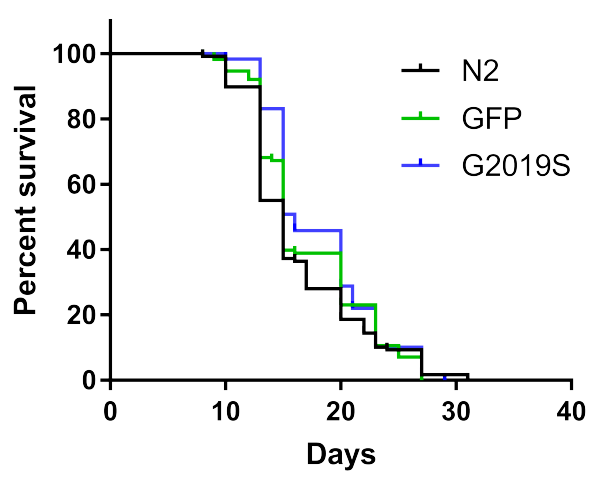

C

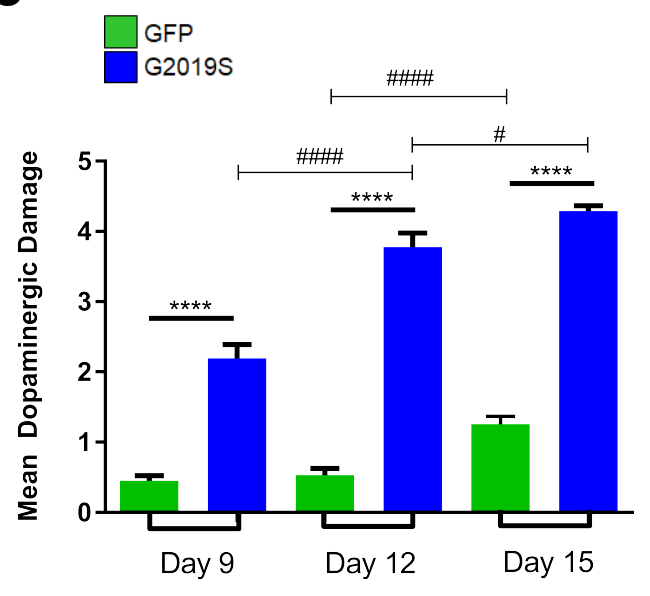

E

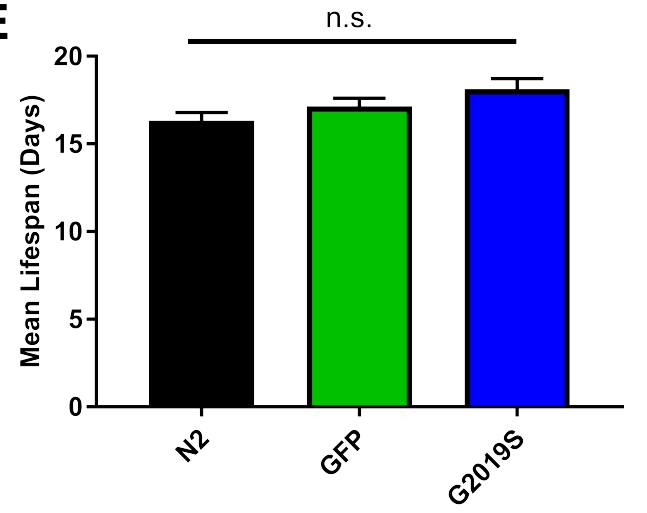

Figure 1 
A
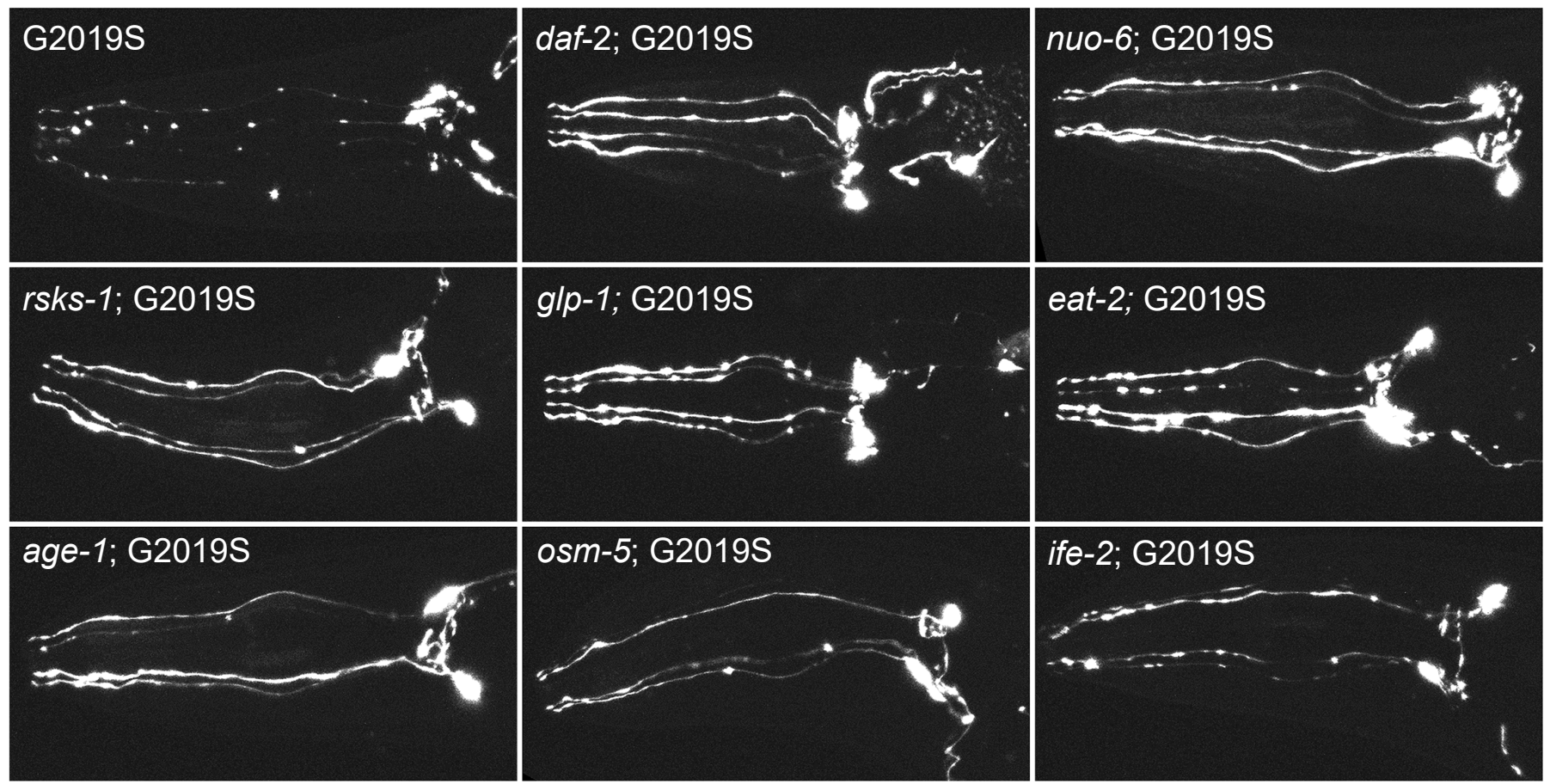

B
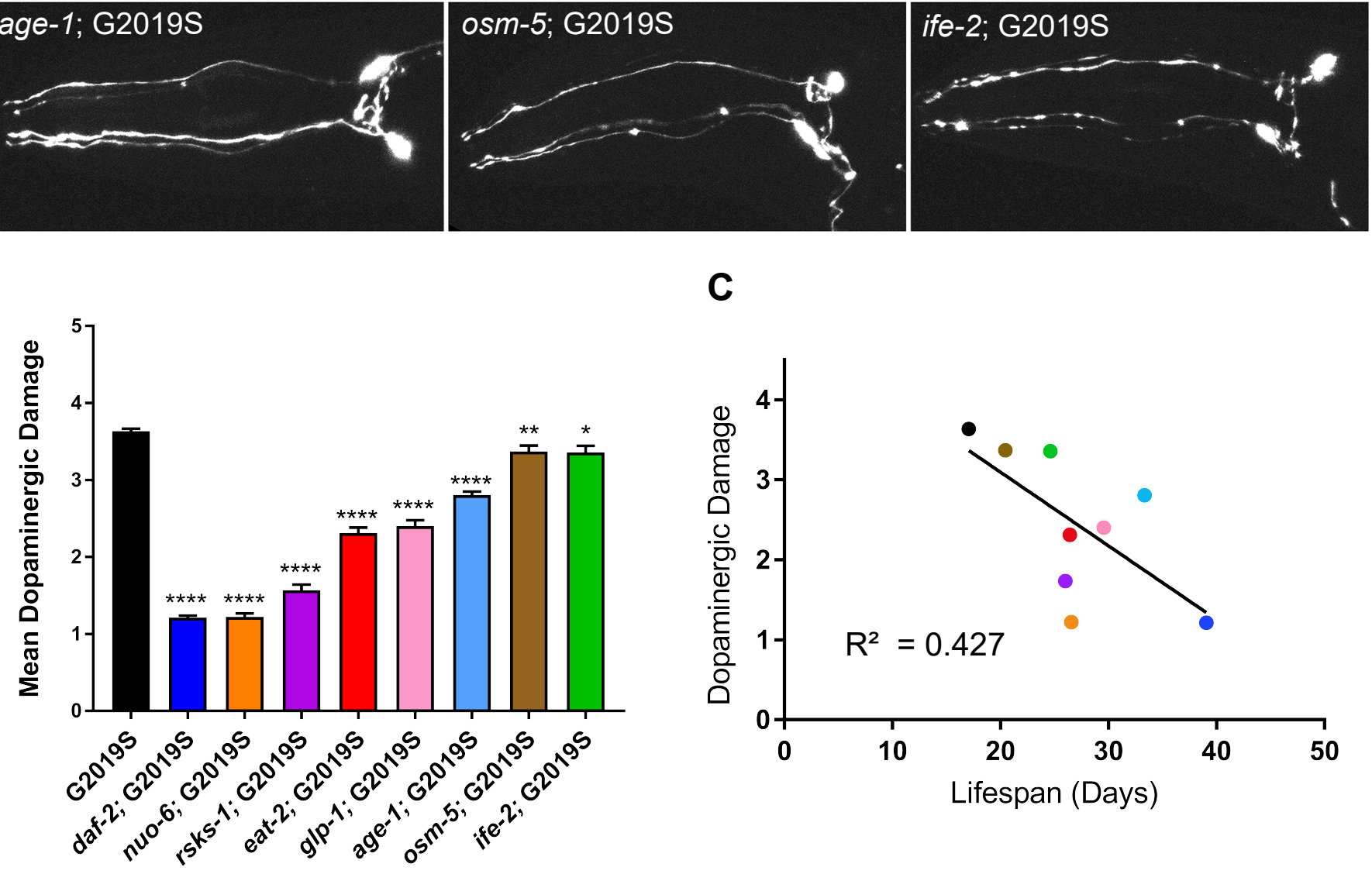

C

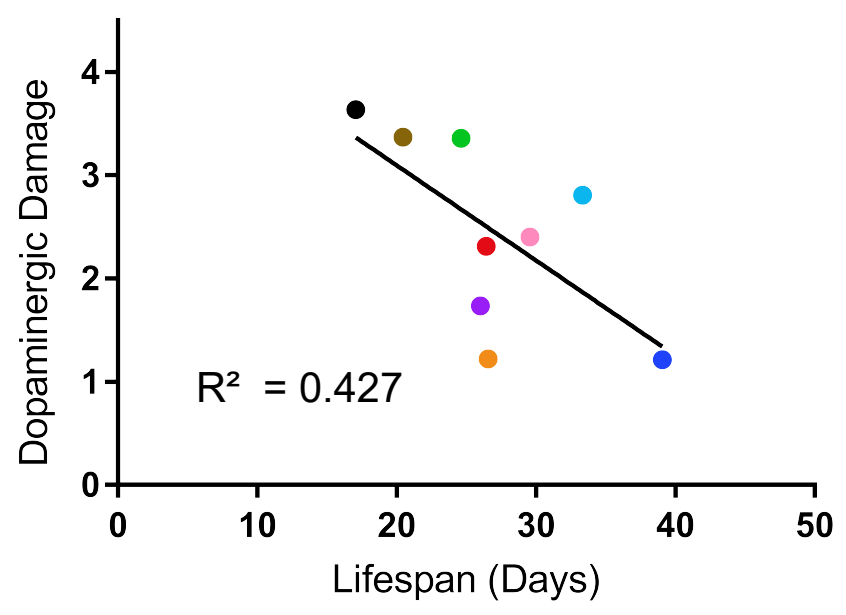

Figure 3 
A

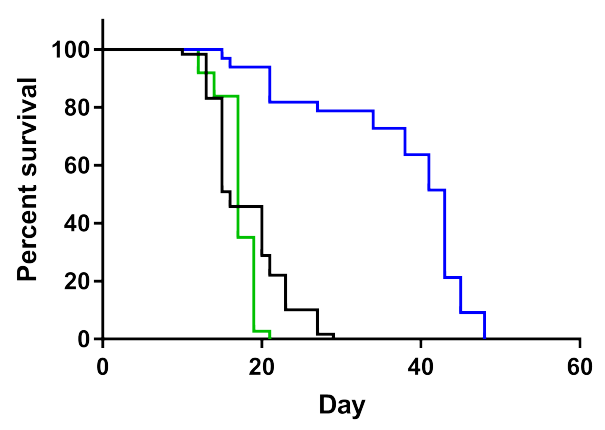

- G2019S

- G2019S; age-1(hx546)

- G2019S; age-1(hx546); daf-16(mu86)
B

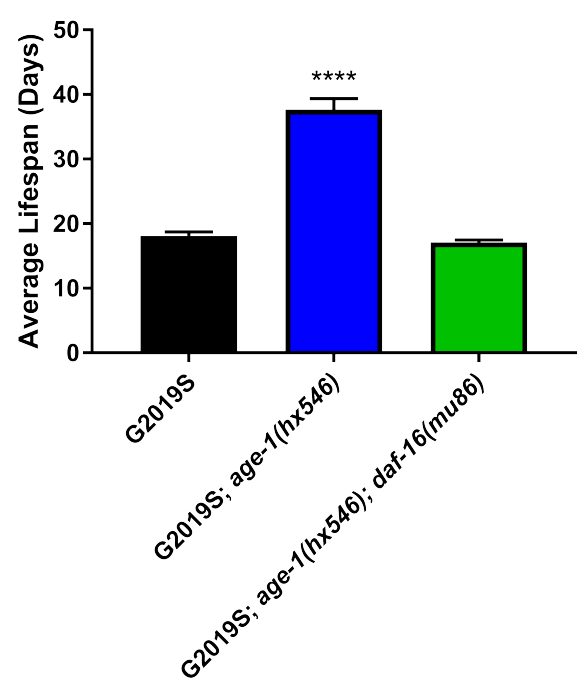

Figure 4
C

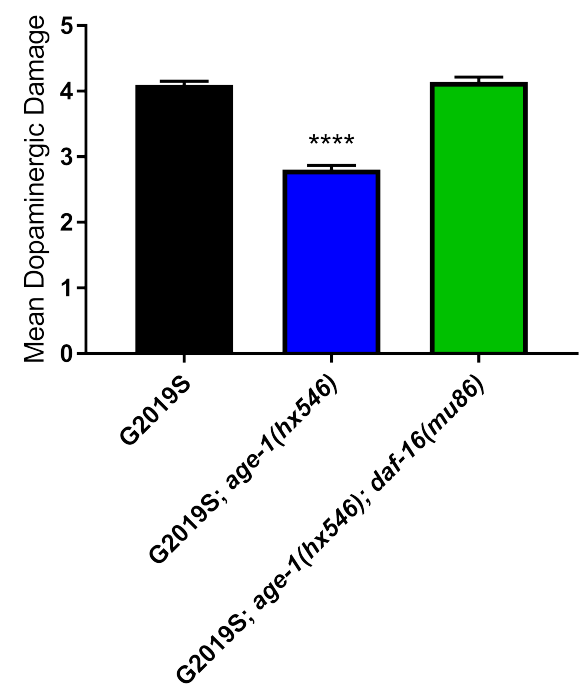




\section{A LRRK2(G2019S) [Systemic]}

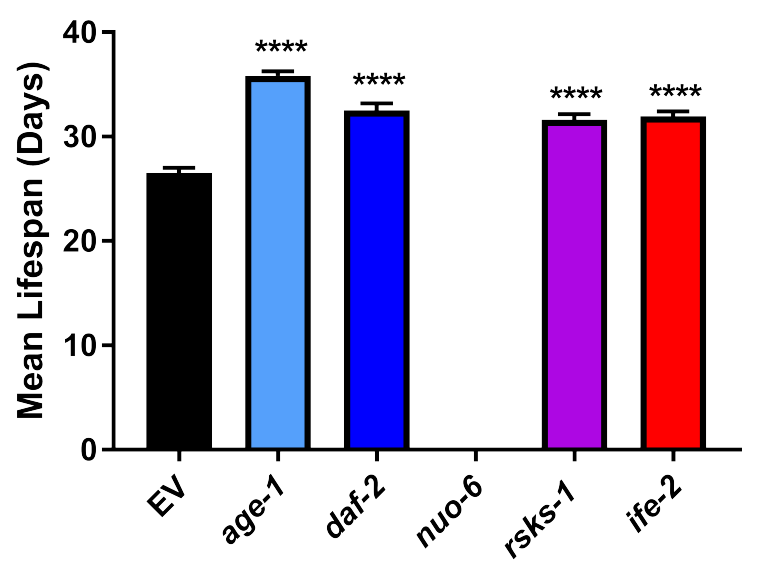

RNAi

C LRRK2(G2019S) [Systemic]

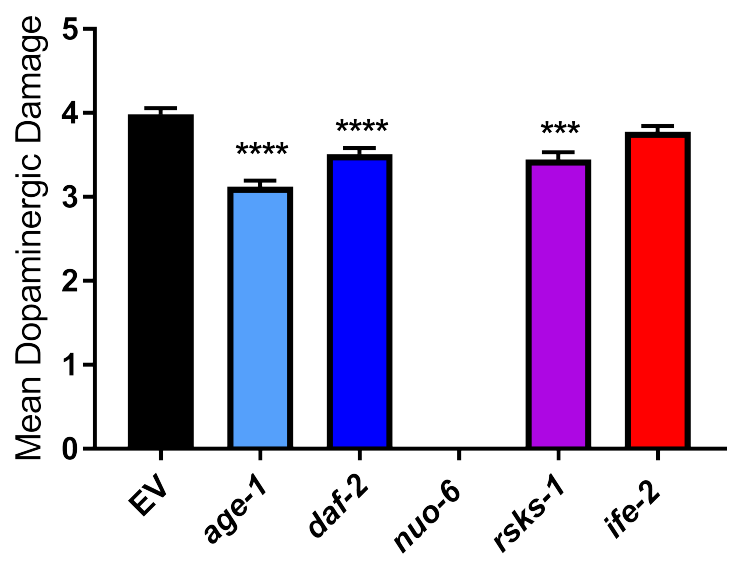

RNAi
B LRRK2(G2019S); SID-1 [Neuron-specific]

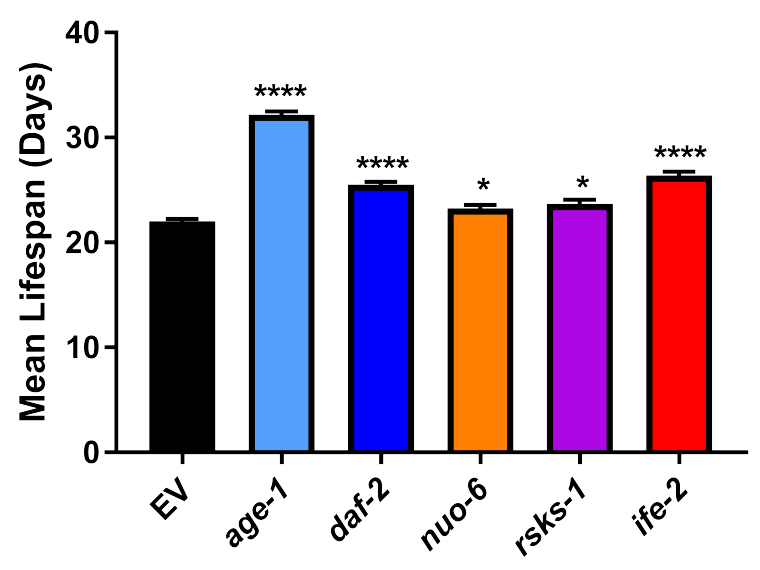

RNAi

D LRRK2(G2019S); SID-1 [Neuron-specific]

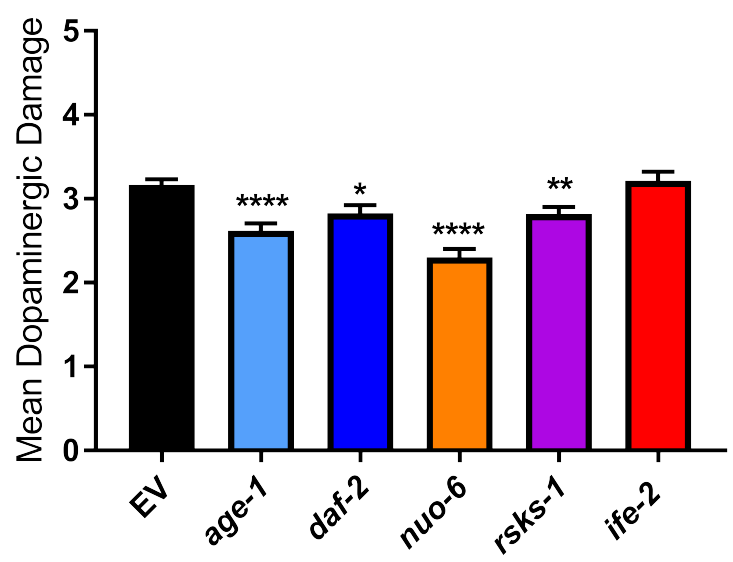

RNAi 


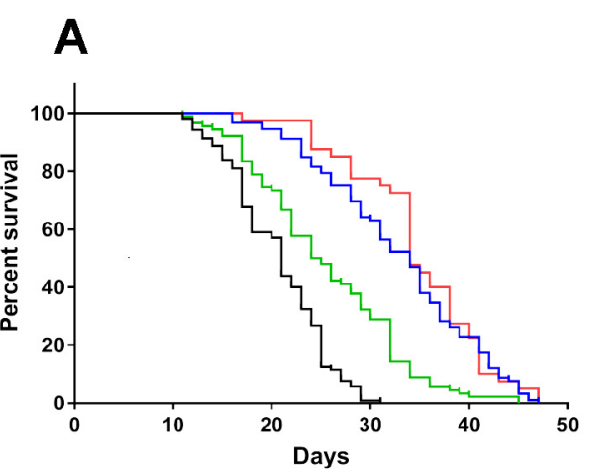

- G2019S

- age-1; G2019S

- age-1; G2019S; pUnc-119::AGE-1

- age-1; G2019S; pDat-1::AGE-1
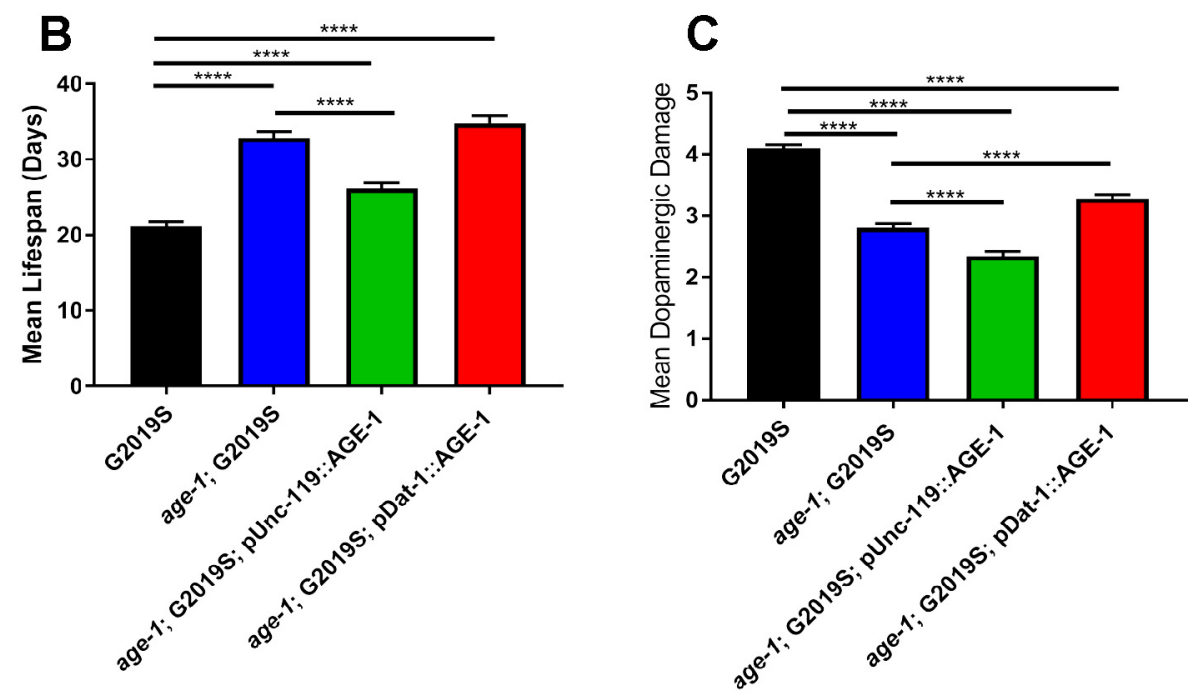

D
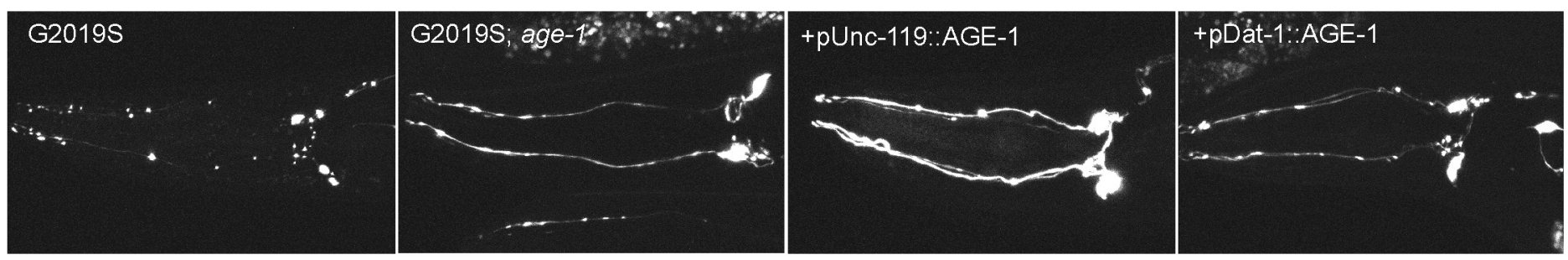

\section{Figure 6}




\section{A}

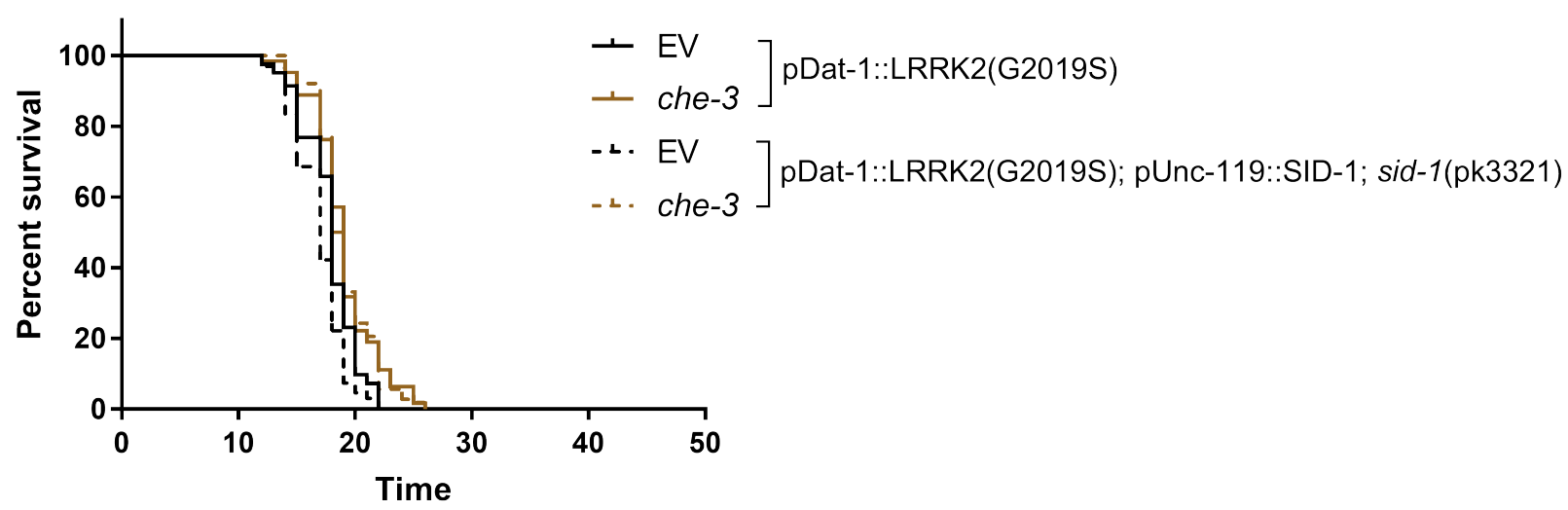

\section{B}

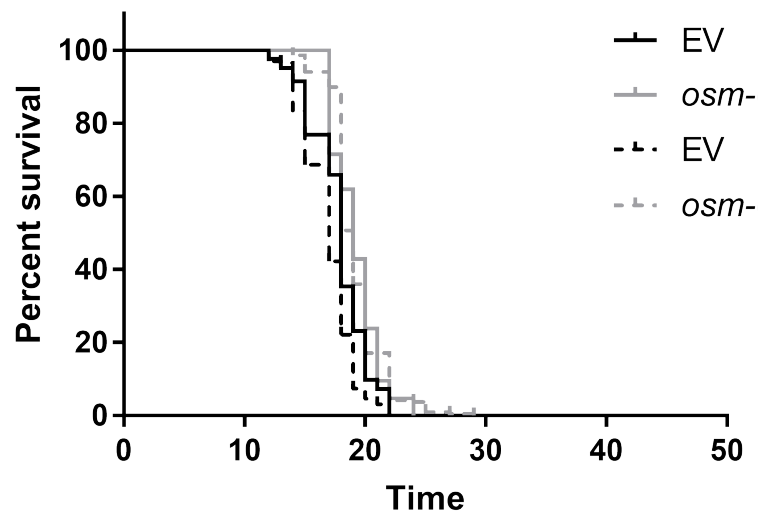

C

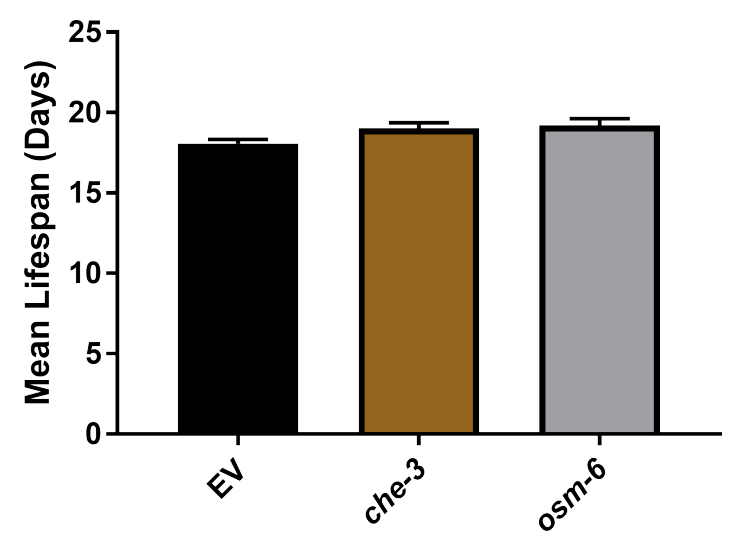

RNAi

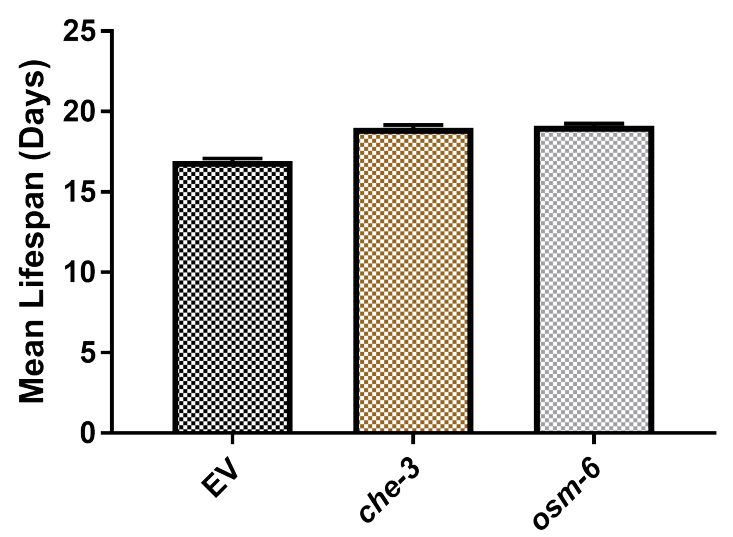

RNAi 
bioRxiv preprint doi: https://doi.org/10.1101/2020.07.06.190025; this version posted July 6, 2020. The copyright holder for this preprint (which was not certified by peer review) is the author/funder, who has granted bioRxiv a license to display the preprint in perpetuity. It is made
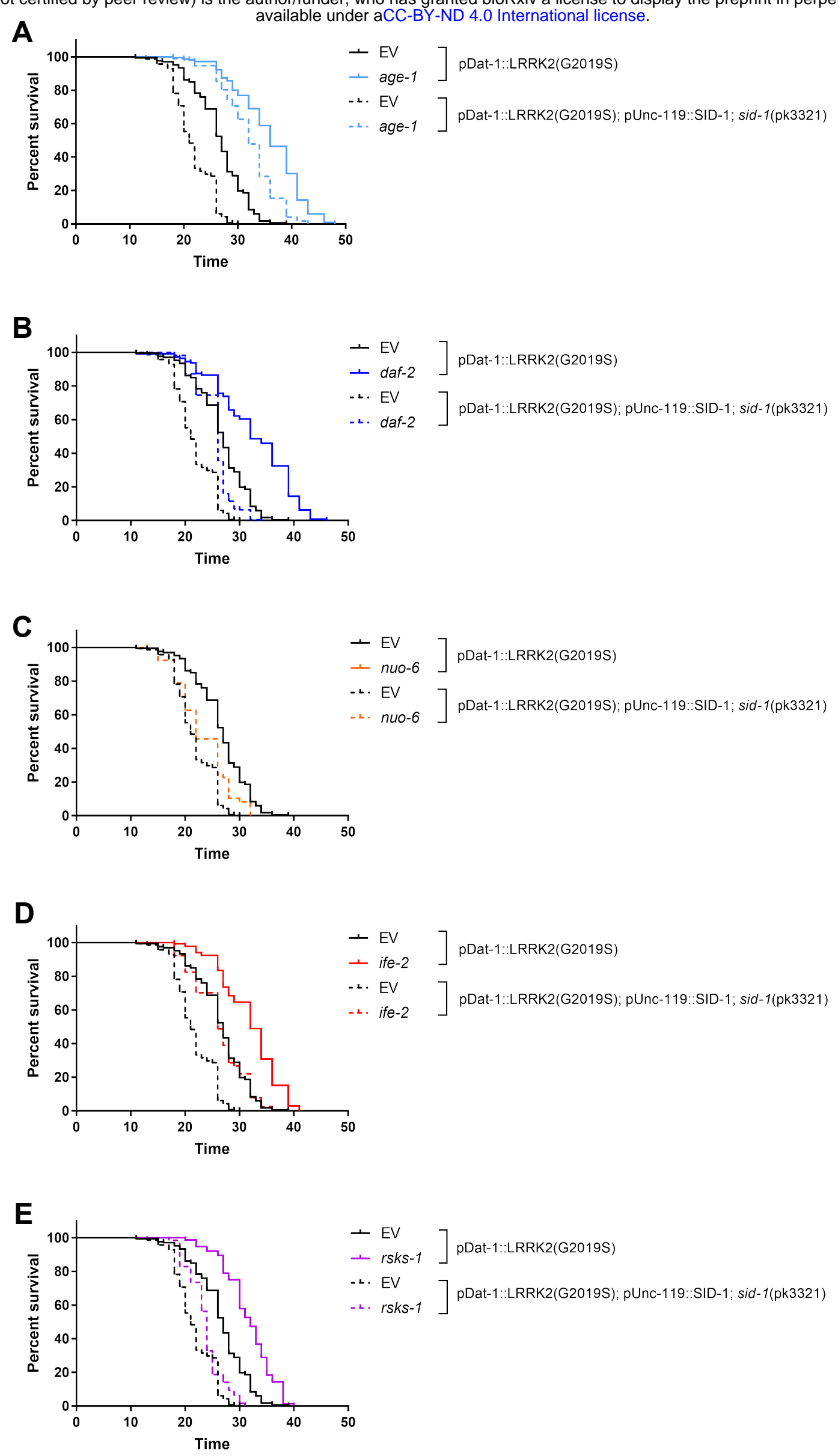
A

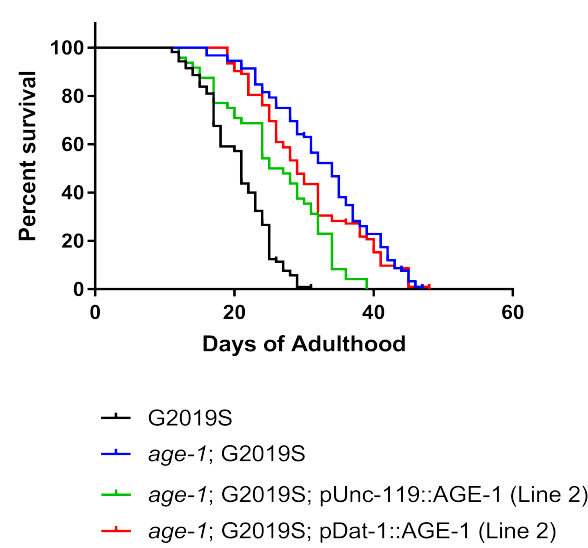

B

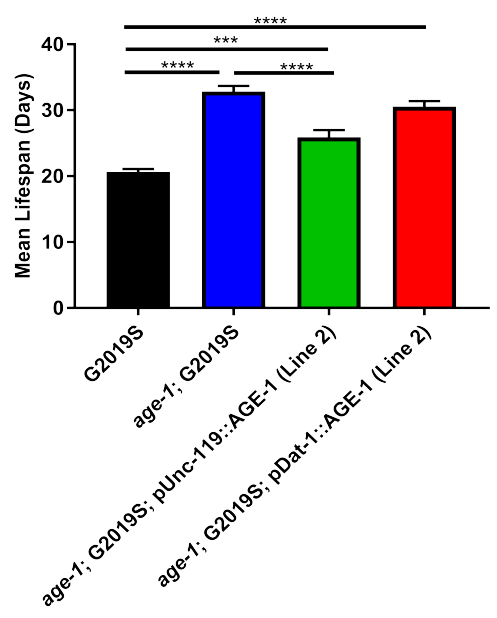

Figure S3
C

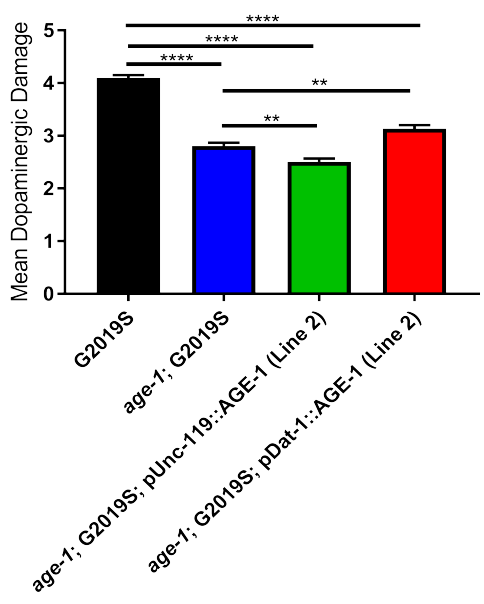

